\title{
Extending the Metal-induced Gap State Model of Schottky Barriers
}

John Robertson ${ }^{1,2,}$, Yuzheng Guo ${ }^{2}$, Zhaofu Zhang ${ }^{1}$, Hongfei Li $^{1}$

1 Department of Engineering, University of Cambridge, Cambridge CB3 0FA, UK

2 School of Electrical Engineering and Automation, Wuhan University, Wuhan, 430072, China

* Email: jr@eng.cam.ac.uk

\begin{abstract}
Fermi level pinning at Schottky barriers strongly limits the minimization of contact resistances in devices and thereby limits the scaling of modern Si electronic devices, so it is useful to understand the full range of behaviors of Schottky barriers. We find that some semiconductor interfaces with compound metals like silicides have apparently weaker Fermi level pinning. This occurs as these metals have an underlying covalent skeleton, whose interfaces with semiconductors lead to mis-coordinated defect sites that create additional localized interface states that go beyond the standard metal-induced gap states (MIGS) model of Schottky barriers. This causes a stronger dependence of Schottky barrier height (SBH) on the metal and on interface orientation. These states are argued to be an additional component needed to extend the MIGS model.
\end{abstract}

\section{Introduction}

It is generally agreed that contact resistances limit the performance of most modern Si electronic devices [1] and of future 2D semiconductors [2]. The reduction of contact resistances would be helped by a simple, more general atomistic model of Schottky barriers. At present, the basic approach is that the Schottky barrier height (SBH) depends on three factors: the metal-induced gap states (MIGS), interfacial point defects, and any interfacial dipole layers [3,4]. MIGS are the evanescent extensions of the metal Bloch states into the semiconductor's band gap. The MIGS model explains how the SBH $\phi_{\mathrm{n}}$, and its variation with metal work function $\Phi_{\mathrm{M}}$, the slope parameter $\mathrm{S}=\partial \phi_{\mathrm{n}} / \partial \Phi_{\mathrm{M}}$, depend on the charge transfer across the interface between the metal Bloch states and the semiconductor gap states [3-9]. The $\mathrm{SBH} \phi_{\mathrm{n}}$ is given by

$$
\phi_{\mathrm{n}}=\mathrm{S}\left(\Phi_{\mathrm{M}}-\Phi_{\mathrm{S}}\right)+\left(\Phi_{\mathrm{S}}-\chi_{\mathrm{s}}\right)
$$

where $\Phi_{M}$ is the metal work function, $\chi_{S}$ is the semiconductor's electron affinity and $\Phi_{S}$ is the semiconductor's charge neutrality level (CNL) with respect to the valence band. The charge transfer across the interface between the metal Bloch states and interface states near the Fermi energy is given by the Cowley-Sze equation [9],

$$
S=\frac{1}{1+\frac{e^{2} N \delta}{\varepsilon \varepsilon_{0}}}
$$

where $\mathrm{N}$ is the density of states at the Fermi level $\left(\mathrm{E}_{\mathrm{F}}\right)$ of MIGS or defects, $\delta$ is their decay length into semiconductor and $\varepsilon$ is the dielectric constant of the semiconductor interface region. $\mathrm{S}$ varies between two extremes, $S=0$ corresponds to strong Fermi level pinning (FLP) (the Bardeen limit) where $\mathrm{N}$ is large and $\mathrm{S}=1$ corresponds to weak FLP (the Schottky limit) where N is small.

The MIGS model has been very successful, particularly in describing the chemical trends of S over a wide range of semiconductors and insulators by replacing Eq. (2) with a simple empirical equation, 


$$
S=\frac{1}{1+0.1\left(\varepsilon_{\infty}-1\right)^{2}}
$$

where $\varepsilon_{\infty}$ is the macroscopic optical dielectric constant $[3,10]$.

However, it is noted that the MIGS model fails to explain several critical factors related to interfacial bonding [11,12]. The effects of dipole layers arising from polar terminations at the interface have already been treated [13,14]. Nevertheless, there are unusual cases of interfacial bonding for silicides, germanides and some interfaces of GaAs [15-18] where the mechanisms are unclear. Here, we show over a range of cases that these involve unusual localized defect gap states created by mis-coordinated sites, so that the gap states vary with face orientation and cause an apparent weakening of Fermi level pinning.

Technologically, a weaker FLP could be very beneficial for semiconductor devices as it would allow us to tune SBHs to minimize interface contact resistances [2,19-22], which are a major limit of both modern CMOS devices and future 2D semiconductor materials. Weaker FLP would allow us to shift SBHs simply by varying the contact metal, without resorting to increased dopant densities to shorten the SB contact depletion length, a method which is presently reaching its limits [23]. Thus it is clearly important to understand why MIGS theory does not presently cover weaker FLP at complex interfaces, and to try to extend the model to include these cases in a simple way.

Formally, MIGS are derived from only the valence and conduction band states intrinsic to the semiconductor. A reference energy, the CNL energy is the branch point of these states and it is the energy where the integral of the density of states equals zero [8],

$$
G(E)=\int_{B Z} \int_{-\infty}^{\infty} \frac{N\left(E^{\prime}\right) d E^{\prime}}{E-E^{\prime}}=O
$$

If the MIGS have a basis of only intrinsic semiconductor states, then the pinning factor $\mathrm{S}$ would be independent of the type of metal (whether elemental metal or compound metal). Also, the SBH would not depend on the face orientation for an isotropic semiconductor like $\mathrm{Si}$.

Thus, the MIGS model fails to explain the orientation dependence of $\mathrm{SBHs}$ for epitaxial $\mathrm{NiSi} / \mathrm{Si}$ interfaces [15,16] or for germanide/Ge interfaces [17], the termination dependence of (Sc, Er)As /GaAs interfaces [18] or the weak pinning at the $\mathrm{Bi} / \mathrm{Si}$ interfaces [24,25]. These various interfaces all show weaker FLP (larger S) and an orientation dependence of SBHs [15-18], which cannot be described by the basic MIGS model. We show that these systems all possess localized defect interface states around $\mathrm{EF}_{\mathrm{F}}$.

Experimentally, silicide contacts are a major exception to this MIGS behavior, in that their slope parameter $S$ is much larger for silicides than for elemental metals on Si [26], see Fig. 1(a). In addition, the calculated and experimental SBHs for (100), (111) and (110) silicide faces are very different [16,27,28], as seen in Fig. 1(b). The different experimental SBHs of the A and B stacking sequences [15] of the epitaxial (111) NiSi2/Si interfaces are well-known, but there is also the large difference between the SBHs of their (100) and (111) faces [16]. These differences are also seen in the equivalent cases of germanide SBH interfaces on Ge [17]. 
These various effects are well reproduced by density functional theory (DFT) supercell calculations [27,28] as given in Fig. 1(b), indicating that this behavior arises from bonding differences, rather than the quality of the contacts. However, the differences are not due to interfacial dipoles or polar bonding, as the Ni-Si bond is essentially non-polar [27]. In order to understand how these differences occur, we have studied the wavefunctions around $\mathrm{E}_{\mathrm{F}}$ for $\mathrm{NiSi} / \mathrm{Si}$ and other silicide and arsenide interfaces using DFT supercell calculations as described below.

\section{Methods}

Our calculations are carried out using the plane wave density functional code CASTEP [29]. We use the generalized gradient approximation (GGA) for the electron exchange-correlation functional, and norm-conserving pseudopotentials with a cutoff energy of $750 \mathrm{eV}$. The convergence is carried out to an energy below $10^{-5} \mathrm{eV}$ per atom, and with forces below $10^{-3} \mathrm{eV} / \mathrm{A}$. A k-point mesh of $4 \times 4$ $\times 2$ is used for Brillouin zone integrations. As the GGA can give a zero band gap for Ge, our density of states calculations for Ge used a different pseudopotential generated by OPIUM code [30] which gives a band gap even in GGA. The GGA functional underestimates the semiconductor band gap, so it will also under-estimate the SBH. We can correct this effect by using hybrid density functionals [31]. Generally though, we are interested in SBH changes, due to the rate of change of SBH with work function or face, which are not affected by the absolute band gap.

The elemental metal work functions are taken from Michaelson [32]. The silicide work functions were calculated by separate density functional supercell calculations on non-polar surfaces with a thick vacuum layer, Fig. 6 . The surface slabs were calculated with frozen geometries, as the surfaces may reconstruct due to the underlying covalent bonding, and this affects their work function. The work function values differ slightly from the geometric average of Si and the metal, as used by Freeouf [26].

\section{Results and discussion}

We first consider the simpler case of the strong orientation dependence of SBHs for the rare-earthAs/GaAs interfaces [18,33-35], as in Fig. 2. The ErAs/GaAs interfaces were studied experimentally because of their good lattice matching. Here, we choose the YAs/GaAs system rather than ErAs/ GaAs for computational ease, to avoid the higher costs of treating the f-states of Er atoms [35].

YAs and other lanthanide arsenides have two phases. The metallic rocksalt phase of YAs is $0.3 \mathrm{eV}$ more stable than the semiconducting zincblende phase. Rocksalt YAs forms a lattice-matched interface with zincblende GaAs, in which the As sublattice is continuous [35]. There are two possible interfaces for a (100) orientation of GaAs, the Ga-terminated and As-terminated interfaces [18,35] shown in Fig. 2. Experimentally, these terminations have a large difference in SBHs, with the SBH of the Ga-terminated interface lying $\sim 1.0 \mathrm{eV}$ higher in the GaAs gap than for the As-terminated case. DFT calculations of the local density of states confirm this, shown in Fig. 3(a). More details of the calculations are found elsewhere [36].

The origin of the different SBH values for different terminations is the characteristic 'defect interface states'. The localized nature of the defect states for YAs/GaAs (100) interfaces is seen in the orbitals shown in Fig. 4. Two different views are provided for each termination. The defect states arise because of an abrupt change in bonding type from the zincblende to the rocksalt lattice. Although the As sublattice is continuous, the interfacial bonding creates mis-coordinated sites on which the defect states are localized.

For the As-terminated (100) interface, the GaAs side has a layer of planar 3-fold As sites. The defect orbital is seen to be localized on the p-like orbitals of the 3-fold As anion sites of this GaAs 
surface layer, and their energies lie near the valence band maximum (VBM) energy of GaAs leading to the small p-type SBH, Fig. 4(a).

For the Ga-terminated interface, the defect states were seen previously, but they are not localized on Ga-sites as said by Lambrecht et al [33]. Rather they lie on the 7-fold As anion interface sites on the YAs side, as seen in the orbital in Fig. 4(b). Although they lie on an anion, the energy of these interfacial As $\mathrm{p}$ states is pushed up to lie around the conduction band minimum (CBM), as seen in the calculated PDOS of this layer, Fig. 3(b). Thus, the YAs/GaAs system is characterized by having localized defect interface states, which cause a Fermi level pinning at different energies for the two different terminations. However, there are not enough arsenide systems to develop any chemical trends in behavior like an $\mathrm{S}$ factor.

We now turn to the silicides, whose SBHs have a stronger dependence on metal work function than elemental metals do, see Fig. 1(a), and whose SBHs show a dependence on the face orientation, unlike elemental metal, see Fig. 1(b). The calculations are carried out on supercells of 9 layers of silicide and 11 layers of $\mathrm{Si}$ (and no vacuum layer) $[28,37]$ to follow the gap states. Lattice symmetry is fixed during the calculation to prevent angular distortions. The lattice geometry was relaxed in GGA. When there is a lattice mismatch between the $\mathrm{Si}$ and the silicide, the x,y lattice constants of the silicide are fixed to those of the $\mathrm{Si}$ and the vertical $\mathrm{z}$ distances are allowed to relax. This roughly conserves the silicide volume.

The NiSi 2 lattice consists of tetrahedrally coordinated $\mathrm{Si}$ sites, continuing the Si-sublattice into the $\mathrm{NiSi}_{2}$. At the (111) interface which is $1 \times 1$, the Ni sites are all 8 -fold coordinated except those at the interface, which are 7-fold, as shown in Fig. 5(a) [27]. The interface is taken to have the A orientation of the two lattices [27]. The (100) interface differs from the (111) case in having a $2 \times 1$ reconstruction, with lateral $\mathrm{Si}-\mathrm{Si}$ bonds and 5-fold $\mathrm{Si}$ sites [38,39] according to high-resolution TEM, as shown in Fig. 5(b).

Our calculations assume the disilicides from $\mathrm{PtSi}_{2}$ to $\mathrm{TiSi}_{2}$ all have the $\mathrm{NiSi} / \mathrm{Si}(111)$ structure with the A stacking sequence [37]. The silicides of metals with larger atomic radii and lower work function from $\mathrm{Zr}$ upwards are taken to adopt the hexagonal $\mathrm{YSi}_{2}$ layered structure [40] (Fig. 5(d)). These interfaces are formed by matching the (0001) silicide faces onto the (111)Si faces as in [28]. The (100) interfaces are also given in ref [28].

We see there is a strong slope of SBH with silicide work function, as shown in Fig. 1, different to that found for elemental metals on Si. The calculated slope is seen to be different for the (111), (110) and (100) faces. This was not seen before, as other groups had not studied a range of silicides.

The resulting interface states around $\mathrm{EF}_{\mathrm{F}}$ are compared in Fig. 7. For interfaces of elemental metals, the states follow those expected from the MIGS model, with continuous Bloch states on the metal side, connecting to decaying MIGS within the semiconductor gap [41], Fig. 7(a). Turning to the silicide interfaces for (111) $\mathrm{NiSi}_{2} / \mathrm{Si}$, for certain energy ranges MIGS are also seen. However, calculations find gap states localized near the interfacial 7-fold interfacial $\mathrm{Ni}$ site ( $\mathrm{Ni}$ 'dangling bond'), Fig. 7(b). These interface states decay on both the semiconductor side and metal side of the interface, very different to the MIGS decay for an elemental metal in Fig. 7(a).

Calculations also show gap states near $\mathrm{E}_{\mathrm{F}}$ for the (100) interface localized around the lateral $\mathrm{Si}-\mathrm{Si}$ bonds and 5-fold coordinated $\mathrm{Si}$ sites, Fig. 7(c). These interface states decay on both the semiconductor side and metal side of the interface, as at (111) $\mathrm{NiSi}_{2} / \mathrm{Si}$ case. 
These anomalous gap states were previously seen by Das [42], Ossicini [43] and Fujitani and Asano [44]. The states were called 'defect states' in Ref. [43]. The state is 'defect-like' in that it decays away from the interface region in both directions. However, they occur for every interface atom in a periodic fashion. Thus, these more complex interfaces possess three types of states: (1) the metal travelling-wave states on the metal side, (2) the MIGS-like states on the semiconductor side, and finally (3) the 'defect-like' states at the interface, localized in both directions. The third state is a new type and not present in the original MIGS model. We find that these states are a general feature of systems that show a depinning effect and the face dependence of SBH.

Fig. 8 plots the gap states for various silicide interface systems. These plots show that the defect gap states add additional density with a peak around $\mathrm{E}_{\mathrm{F}}$ for the (111) and (100) interfaces. The energy of these peaks differs for each face. The peak energy is plotted against the silicide work function in Fig. 9. The peak energy is seen to follow the same variation as that of the SBH in Fig. 1(b). Thus, this extra DOS determines the EF pinning. The defect states dominate the effect of the underlying MIGS in fixing $\mathrm{EF}_{\mathrm{F}}$ for each metal silicide. The defect states have a different shape for each face orientation, and this causes the orientation dependence of SBH.

We noted earlier that the slope $S$ was steeper for silicides than for elemental metals. From Eq. (2), a larger S would imply a lower gap DOS. A lower DOS would be very beneficial if this actually occurs. However, Fig. 8 shows this is not what happens, the DOS goes up. Silicides have an underlying background covalent lattice. When they form interfaces with covalent semiconductors, their skeletons do not perfectly match, and this creates a periodic array of coordination defects. These defects create the localized interface states. These states have a larger DOS than the basic MIGS, and their DOS pulls $E_{F}$ across the gap as the transition metal varies, along a staircase of transition metal silicide energies. This is how the apparent 'Fermi level depinning' arises. But it does not arise from simply clearing the gap states, as is apparent in Fig. 8. The classic case of Fermi level depinning occurs for defect passivation at interfaces [45]. But chemically varying the transition metal in a silicide passivates no defects, it just replaces one metal for another with a different energy.

Overall, although classic MIGS are semiconductor-only states, at complex interfaces such as the silicides, the interfacial defect states create a strong energy dependence of the pinning energy as the silicide's metal varies. The shape of the defect states also varies with interface orientation, which causes the SBH to vary with orientation.

The silicides are a relatively unique class of metalloids, which can form with metals of many valences, and wide range of work functions. They have an underlying covalent skeleton whose interfaces with Si create coordination defects. Their interface Fermi levels with Si appear to be less pinned but are not. Silicides are unusual in that there are so many silicides whose work functions span a large part of the Si gap. The interfacial defects seen here are part way to the distributed point defects that provide stronger pinning in other systems [44].

The third related system possessing interfacial 'defect states' is the Bi/Si interface. We represent this interface by the $\mathrm{Sb} / \mathrm{Si}$ interface, to avoid the extra computationally complexity of including spinorbit coupling in $\mathrm{Bi}$.

This heterojunction is notable because $\mathrm{Sb}$ is a semimetal that has a very low density of states (DOS) around Ef. Experimentally, Bi contacts on Ge were found to be ohmic [24], and this is partly attributed to the low DOS at $\mathrm{E}_{\mathrm{F}}$ which provides only weak pinning of metal $\mathrm{SBHs}$ on $\mathrm{Bi}$ or $\mathrm{Sb}$, as shown in Fig. 10(a). 
The slope factor of SBH for different metals contacting Sb is calculated in supercells. It is found to be much larger than that expected from its dielectric constant [2], according to Eq. (3), Fig. 10(b). We have modeled the interfaces between $\mathrm{Si}$ and three orientations of the rhombohedral $\mathrm{Sb}(\mathrm{r}-\mathrm{Sb})$ lattice [25]. Table 1 shows that the SBHs vary quite strongly with interface orientation, as they do in the silicides and arsenides.

Figs. 10(c,d) plot the orbitals at EF for each interface. Some localized interfacial 'defect states' are again seen. These localized states differ at each orientation. It is this that causes the SBHs to vary with orientation. However, the states arise from mis-coordinated sites due to the poor lattice match of $\mathrm{Sb}$ and $\mathrm{Si}$. The defect states are less localized compared with silicides or germanides, and their effect on the gap DOS would be less. However because $\mathrm{Sb}$ is a semimetal with a low N(Ef) of MIGS, this magnifies the effect of the defect states and causes the observed weak pinning and face dependence.

Table 1. Calculated p-type SBH for $\mathrm{Sb}$ on $\mathrm{Si}$, measured from VBM of Si.

\begin{tabular}{|c|c|c|c|}
\hline Face & $(100)$ & $(110)$ & $(111)$ \\
\hline SBH (eV) & 0.9 & 0.5 & 0.7 \\
\hline
\end{tabular}

\section{Summary}

In conclusion, we have identified metal silicides, rare-earth arsenides and Sb interfaces as three cases that lie outside the standard MIGS model, where Schottky barrier heights now vary with face orientation and vary with metal work function more than do the equivalent elemental metals. The interfaces are found to contain bonding configurations such as dangling bonds, lateral bonds or miscoordinated sites which create defect gap states, which are difficult to describe within the MIGS basis set. This leads to a localization of states at the interface. These states add to the MIGS density of states and they pull $\mathrm{E}_{\mathrm{F}}$ across the gap, which increases the slope parameter $\mathrm{S}$. This seems to de-pin $\mathrm{E}_{\mathrm{F}}$ but does not do so, as there is a higher DOS overall induced by the defects. The orientation dependence of the SBH S factor is a second signature of apparent de-pinning. Although these states do not cause the classic Fermi level depinning, which removes states from the gap, a specific interface chemistry and its defects can be used to set the Fermi level at the desired band edge, and so in this way reduce the SBH to zero. This is sufficient from a device perspective.

Acknowledgments: The authors acknowledge funding from EPSRC grant EP/P005152/1. 
1. S. Datta, R. Pandey, A. Agrawal, S. K. Gupta and R. Arghavani, Tech Digest VSLI Technol. p978 (2014)

2. A. Allain, J. Kang, and K. Banerjee, A. Kis, Nat. Mater. 141195 (2015)

3. W. Monch, Phys. Rev. Lett. 581260 (1987)

4. J. Robertson, J. Vac. Sci. Technol. B 181785 (2000)

5. J Bardeen, Phys. Rev. 71717 (1947)

6. V. Heine, Phys. Rev. A 138 A1689 (1965)

7. F. Flores, E. Louis, and F. Yndurian, J. Phys. C: Solid State Phys. 6 L465 (1973)

8. J. Tersoff, Phys. Rev. Lett. 52465 (1984); J Tersoff, Phys. Rev. B 32 6968(R) (1985)

9. A. M. Cowley, S. Sze, J. Appl. Phys. 363212 (1965)

10. W. Monch, Appl. Surf. Sci. 92367 (1996)

11. R. T. Tung, Phys. Rev. Lett. 846078 (2000)

12. R. T. Tung, Appl. Phys. Rev. 1011304 (2014)

13. C. Berthod, N. Binggeli, and A. Balderschi, Phys. Rev. B 68085323 (2003)

14. K. Y. Tse, J. Robertson, Phys. Rev. Lett. 99086805 (2007)

15. R. T. Tung, Phys. Rev. Lett. 52461 (1984)

16. R. T. Tung, J. Vac. Sci. Technol. B 111546 (1993)

17. T. Nishimura, T. Yajima, and A. Toriumi, Appl. Phys. Express 9081201 (2016).

18. C. J. Palmstrom, T. L. Cheeks, H. L. Gilchrist, J. G. Zhu, C. B. Carter, B. J. Wilkens, and R. Martin, J. Vac. Sci. Technol A. 101946 (1992)

19. A. Agrawal, N. Shukla, K. Ahmed, and S. Datta, Appl. Phys. Lett. 101042108 (2012)

20. S. Das, H. Y. Chen, A. V. Penumatcha, and J. Appenzeller, Nano Lett. 13100 (2013)

21. Y. Liu, J. Guo, L. Liao, S. J. Lee, M. Ding, I. Shakir, V. Gambin, Y. Huang, and X. Duan, Nature 557 696 (2018)

22. D. Liu, Y. Guo, L. Fang, J. Robertson, Appl. Phys. Lett. 103183113 (2013); Y. Guo, D. Liu, J. Robertson, ACS Appl. Mat. Interface 725709 (2015)

23. Z. N. Weinrich, X. Li, S. Sharma, V. Craciun, M. Ahmed, E.A.C. Sanchez, S. Moffatt, K.S. Jones, Thin Solid Films 6851 (2019)

24. T. Nishimura, X. Luo, S. Matsumoto, T. Yajima, and A. Toriumi, AIP Adv. 8095013 (2019)

25. Y. Guo, H. Zhang, and J. Robertson, Extended abstracts, Solid State Devices and Materials conf (2018) PS1-06

26. J. L. Freeouf, Solid State Commun. 331059 (1980);

27. L. Lin, Y. Guo, and J. Robertson, Appl. Phys. Lett. 101052110 (2012)

28. H. Li, Y. Guo, and J. Robertson, Sci. Rep. 716669 (2017).

29. S. J. Clark, M.D. Segall, C.J. Pickard, P. J. Hasnip, M. J. Probert, K. Refson, M.C. Payne, and Z. Kristallogr. 220, 567 (2005) DOI: 10.1524/zkri.220.5.567.65075

30. A. M. Rappe, K. M. Rabe, E. Kaxiras, and J. D. Joannopoulos, Phys. Rev. B 411227 (1990)

31. S. J. Clark, J. Robertson, Phys. Rev. B 82, 085208 (2010)

32. H. B. Michaelson, J Appl. Phys. 484729 (1977)

33. W. Lambrecht, A. G .Petukhov, and B. T. Hemmelman, Solid State Commun. 108361 (1998)

34. K. T. Delaney, N. Spaldin, and C. Van de Walle, Phys. Rev. B 81165312 (2010)

35. D. O. Klenov, J. M. Zide, J. D. Zimmerman, A. C. Gossard, and S. Stemmer, Appl. Phys. Lett. 86 $241901(2005)$

36. Z. Zhang, Y. Guo, and J Robertson, Appl. Phys. Lett. 116251602 (2020)

37. Z. Zhang, Y. Guo, and J. Robertson, submitted (2020)

38. B. D. Yu, Y. Miyamoto, O. Sugino, A. Sakai, T. Sasaki, and T. Ohno, J. Vac. Sci. Technol. B 19, 1180 (2001)

39. U. Falke, A. Bleloch, M. Falke, and S. Teichert, Phys. Rev. Lett. 92, 116103 (2004)

40. S. Vadre, T. Kalka, C. Preinesberger, M. Dahne-Prietsche, Phys. Rev. Lett. 821927 (1999)

41. S. G. Louie, J. Chelikowsky, and M. L. Cohen, Phys. Rev. B 152154 (1977)

42. G. P. Das, P. Blochl, O. K. Andersen, N. E. Christensen, O. Gunnarsson, Phys. Rev. Lett. 631168 (1989)

43. S. Ossicini, O. Bisi, C. M. Bertoni, Phys. Rev. B 425735 (1990)

44. H. Fujitani, S. Asano, Phys. Rev. B 508681 (1994)

45. J. Robertson, Appl. Phys. Lett. 94152104 (2009) 


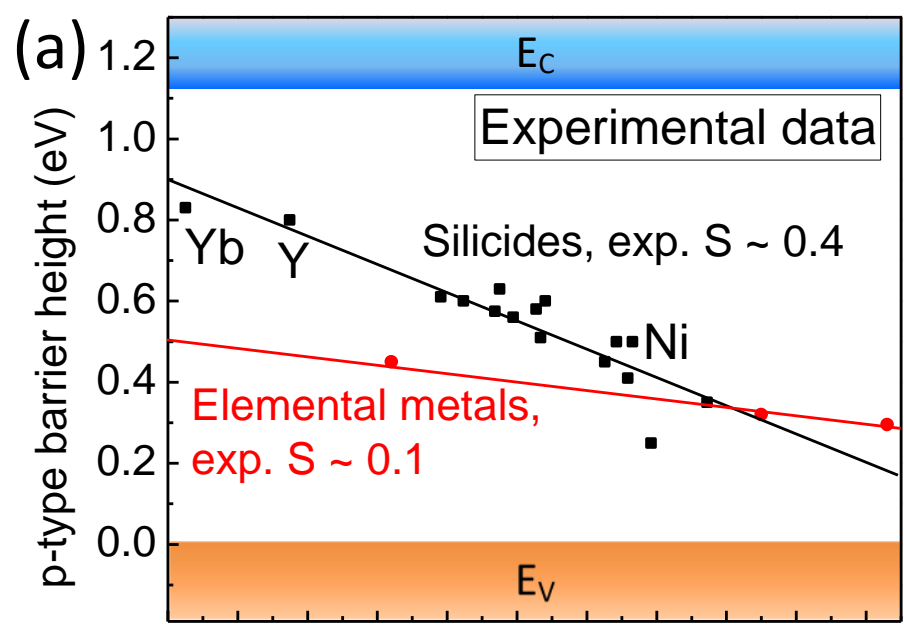

3.6 3.8 4.0 4.2 4.4 4.6 4.85.0 5.25.45.6

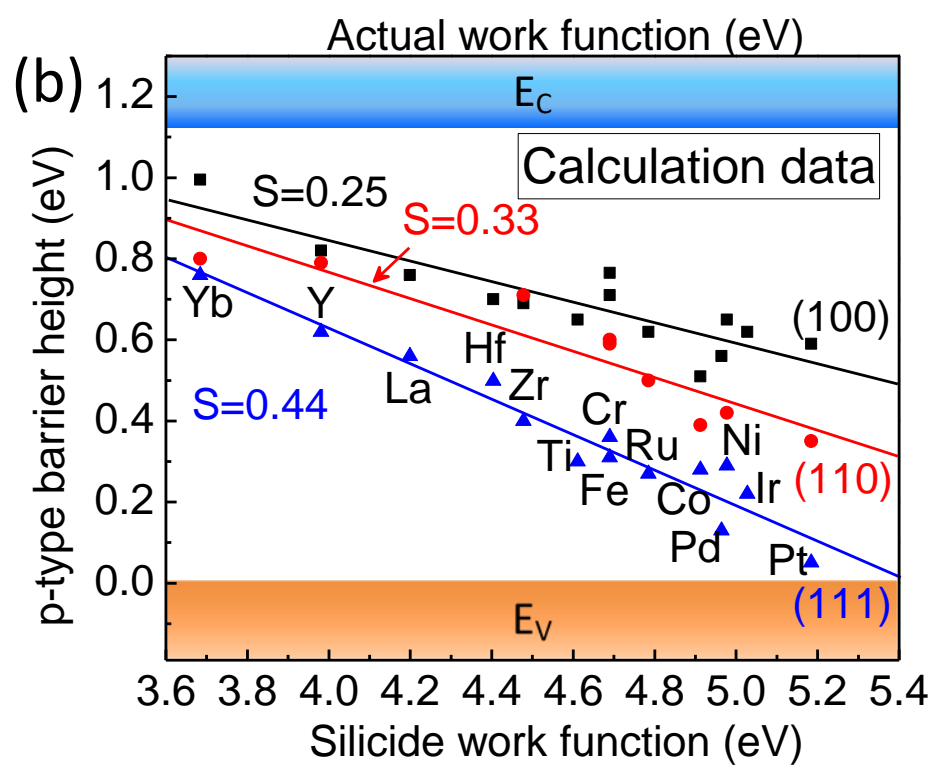

Fig. 1. (a) Comparison of the experimental SBH values for elemental metals and silicides on Si. (b) Calculated SBHs of silicides on (111), (100) and (110) Si faces using supercells, and consistent with experimental data for germanides. The silicide work function is calculated as geometric average of metal and $\mathrm{Si}$.
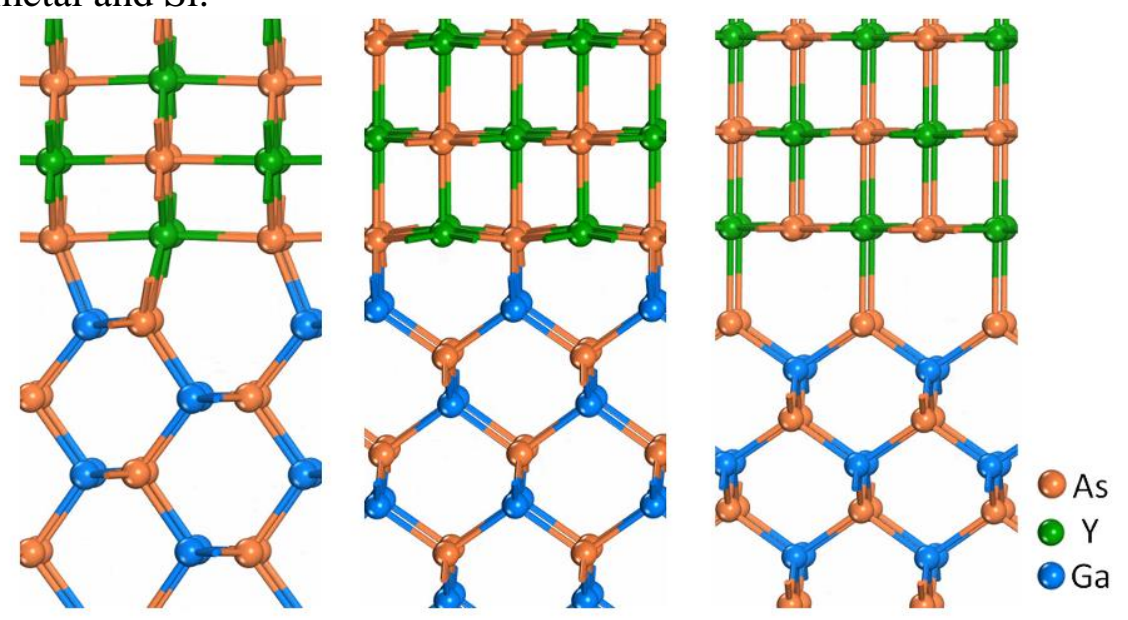

(a) (110) interface (b) (100) Ga-term. (c) (100) As-term.

Fig. 2. Illustration of the interface structures of non-polar (110), Ga-terminated (100) and (c) Asterminated (100) YAs/GaAs interfaces viewed for the (110) plane. 

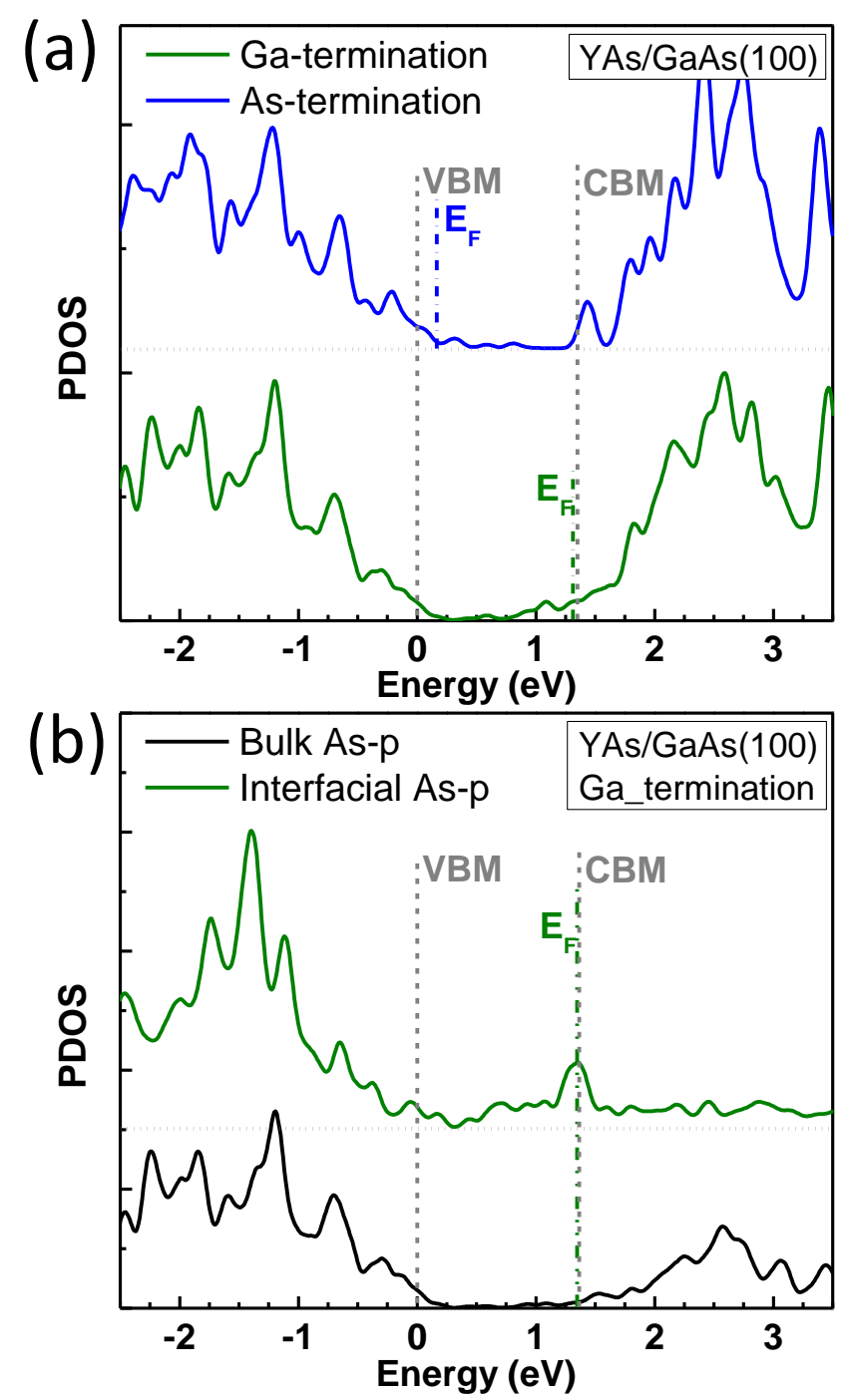

Fig. 3. (a) Calculated density of states for layers in the (100)YAs/GaAs interface system of bulk atoms that are away from the interfacial region, showing large difference in Fermi energies (i.e., SBHs) for the Ga- and As- terminations. (b) Calculated PDOS for As sites on the last YAs layer of YAs side, of Ga-terminated YAs/GaAs(100) interface. 

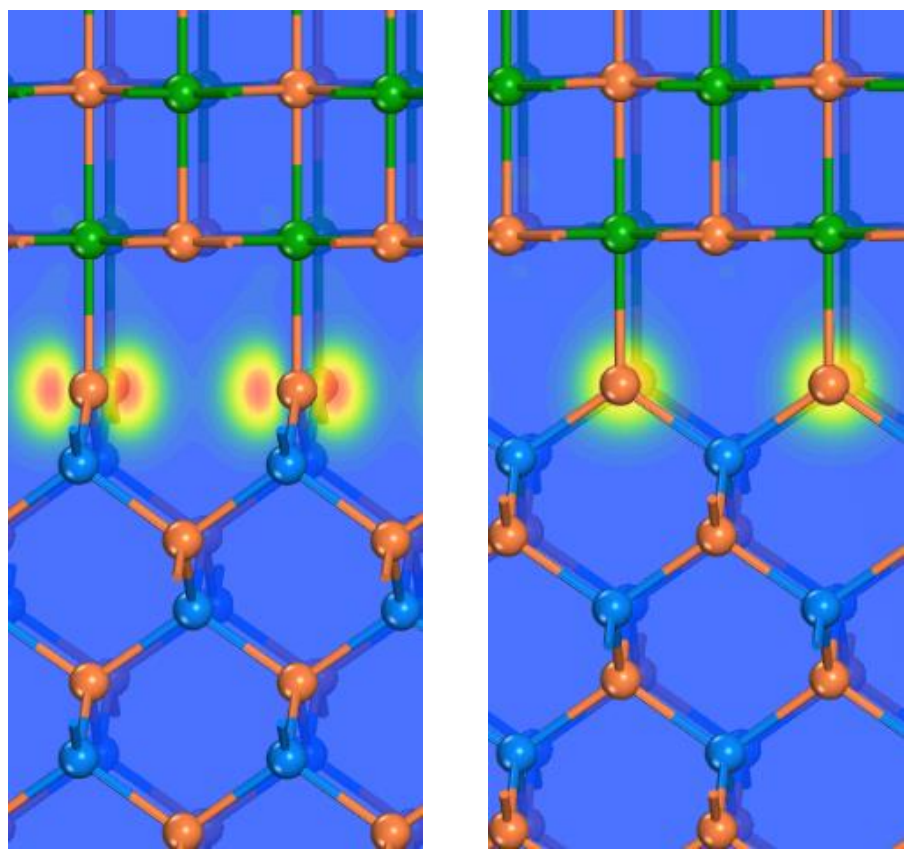

(a) YAs/GaAs (100) As-terminated
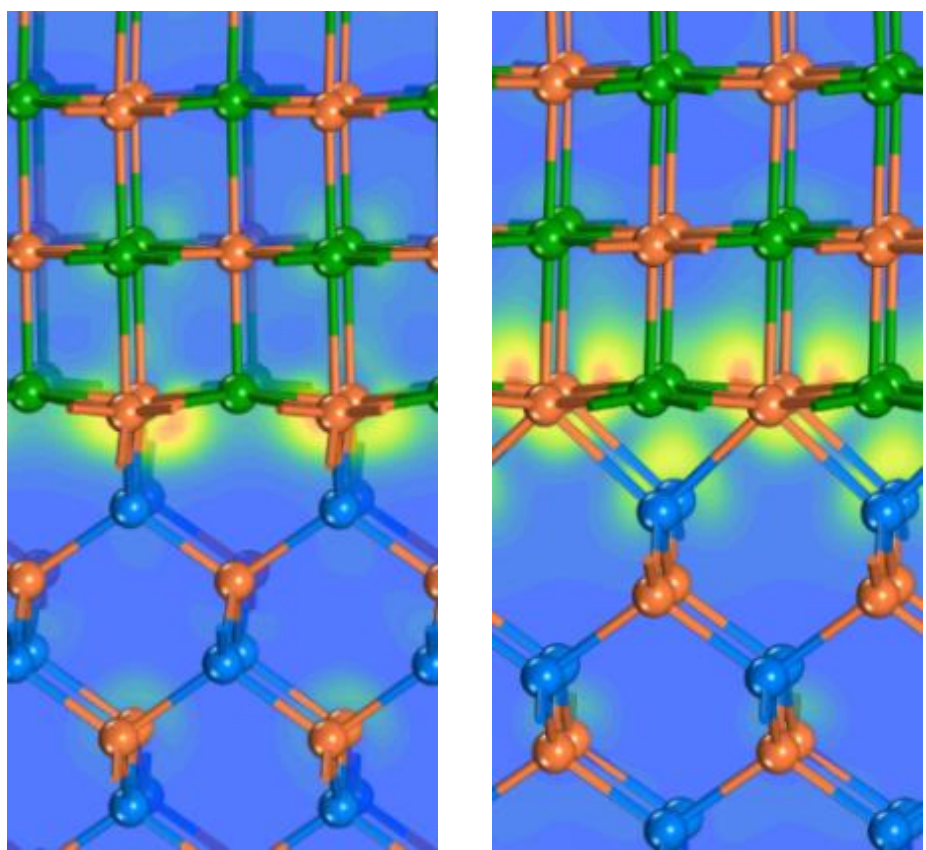

(b) YAs/GaAs (100) Ga-terminated

Fig. 4. Lattices and wavefunctions at Fermi energy for the (a) As-terminated and (b) Ga-terminated YAs/GaAs (100) interfaces viewed for the (110) plane. 


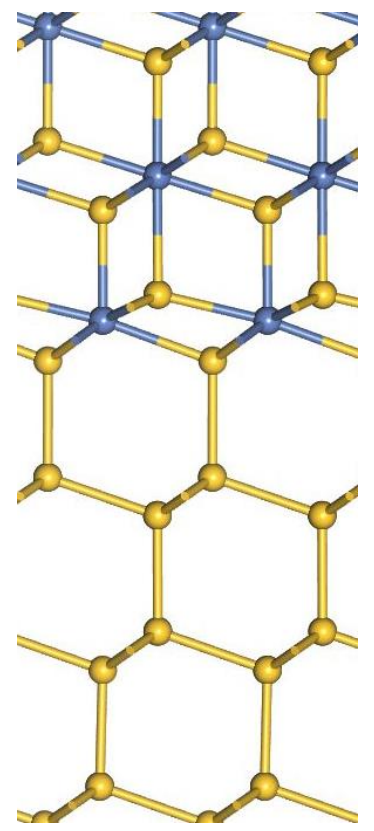

(a) (111)

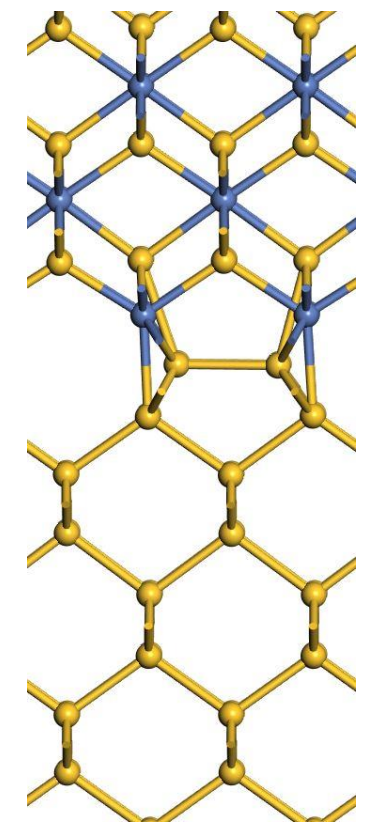

(b) (100)

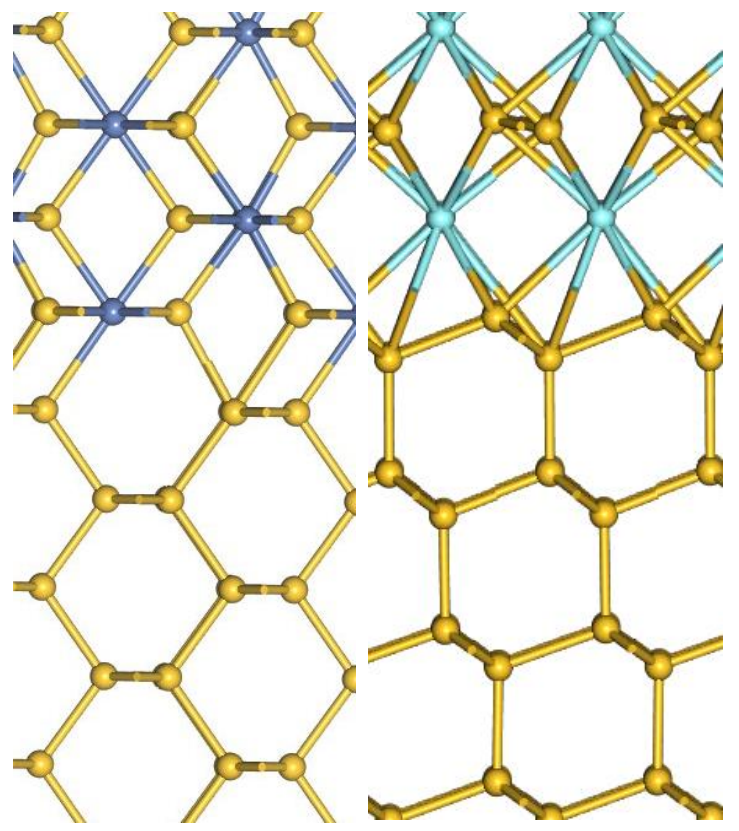

(c) (110)

(d) $\mathrm{YSi}_{2} / \mathrm{Si}(111)$

Fig. 5. Four interfaces of silicides/Si. (a) The $1 \times 1$ interface of $\mathrm{NiSi} / 2 \mathrm{Si}(111)$. (b) the $2 \times 1$ dimerized interface of $\mathrm{NiSi}_{2} / \mathrm{Si}(100)$. (c) a reconstructed interface of $\mathrm{NiSi}_{2} / \mathrm{Si}$ (110). (d) the $\mathrm{YSi}_{2} / \mathrm{Si}_{(111)}$ interface.

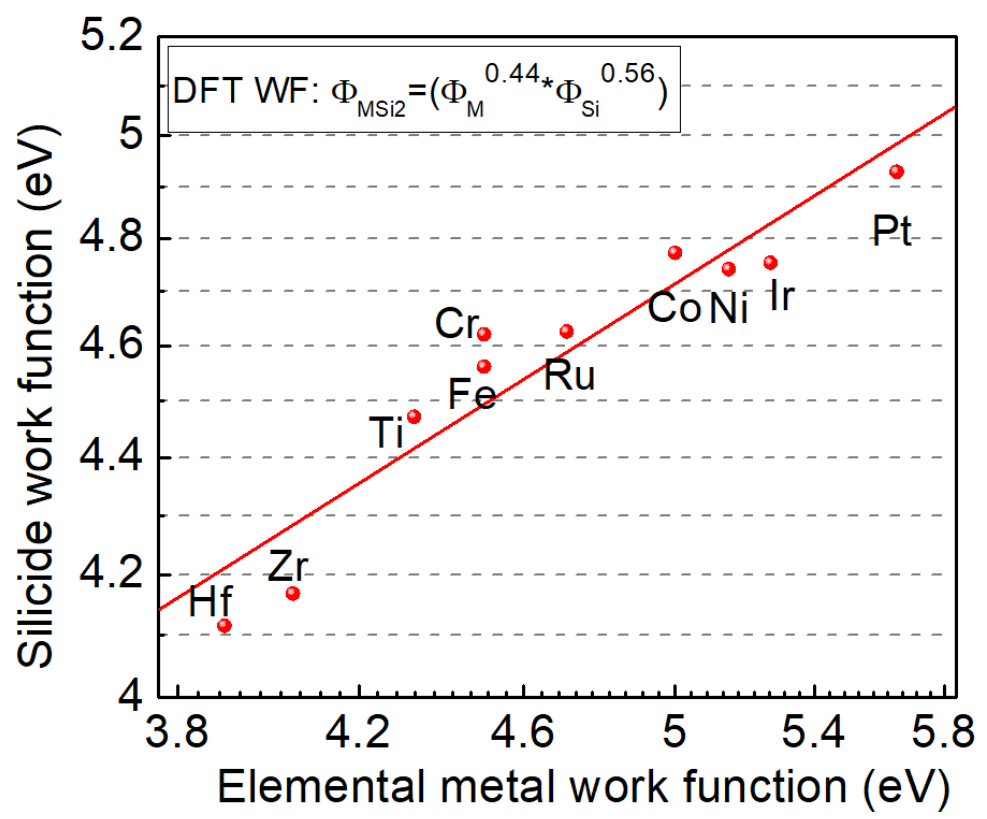

Fig. 6. Variation of GGA calculated work function of silicides versus the parent metal work function. The silicide work function formula is inserted, with a power of 0.44 for metal with the logarithmic fitting. 


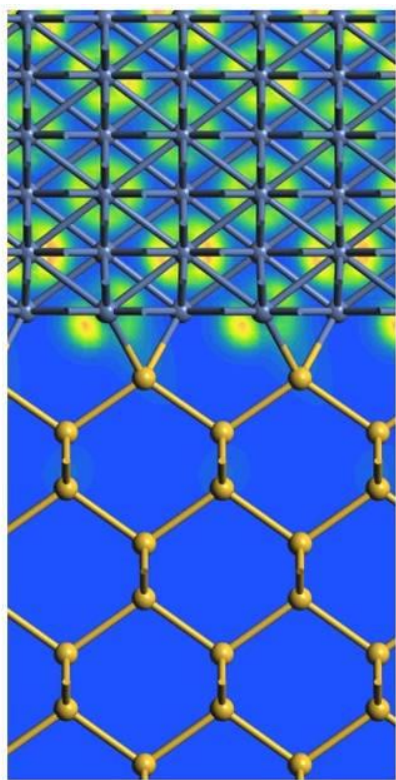

(a) $\mathrm{Ni} / \mathrm{Si}(100)$

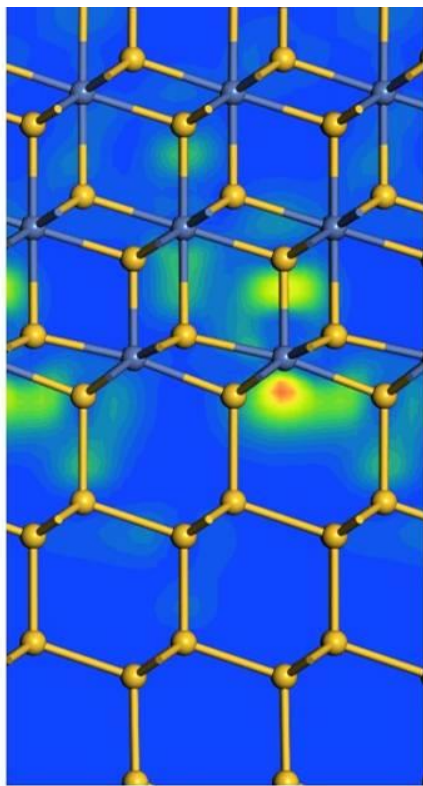

(b) $\mathrm{NiSi}_{2} / \mathrm{Si}(111)$

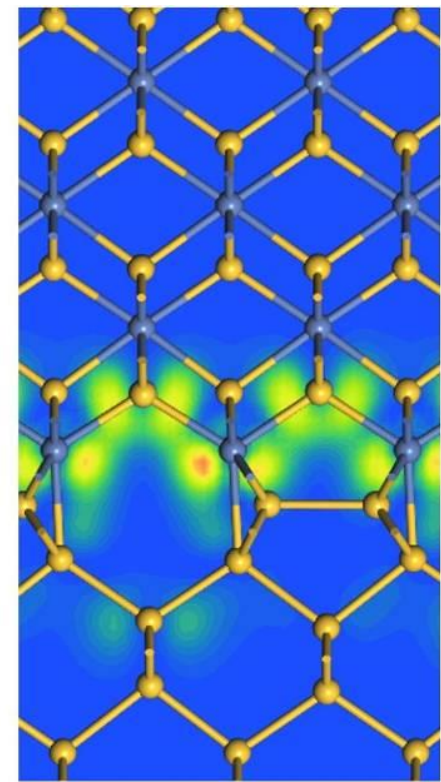

(c) $\mathrm{NiSi}_{2} / \mathrm{Si}(100)$

Fig.7. Wavefunction around Fermi level for (a) Ni/Si(100) with MIGS only, (b) NiSi2/Si(111) with MIGS and defects, and (c) $\mathrm{NiSi}_{2} / \mathrm{Si} 2 \times 1(100)$ interfaces with MIGS and defects. The silicides (b,c) show localized states decay on both directions from the interface. The MIGS in (a) show no decay on metal side.
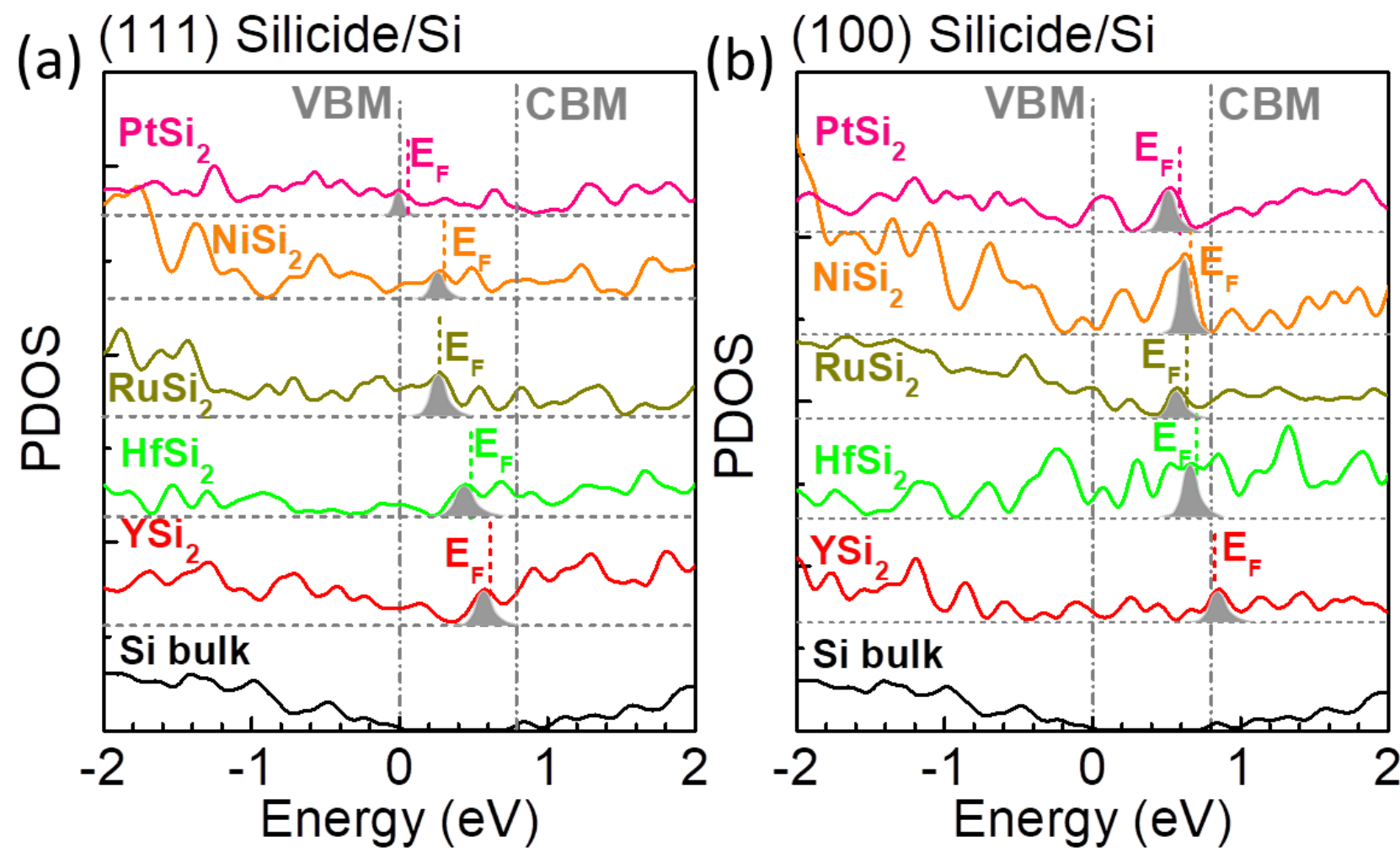

Fig. 8 (a) Gap state PDOS of interfacial layers of various silicides on (111)Si showing the peak due to localised defect states. (b) similar for (100). 


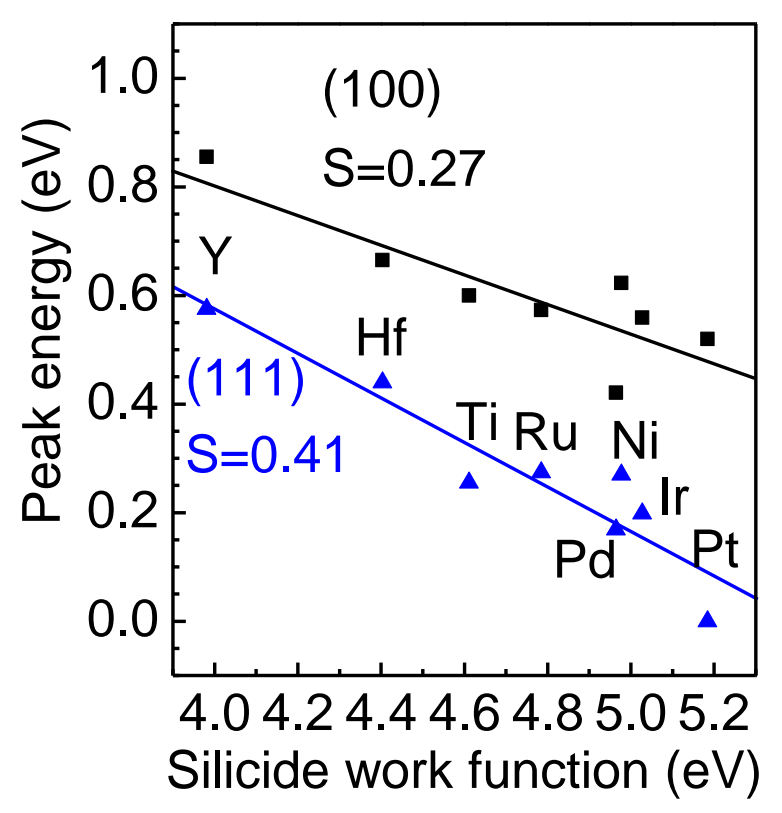

Fig. 9. Variation of peak energy of defect states from Fig. 7(a,b) vs. silicide work function, showing different slope values.
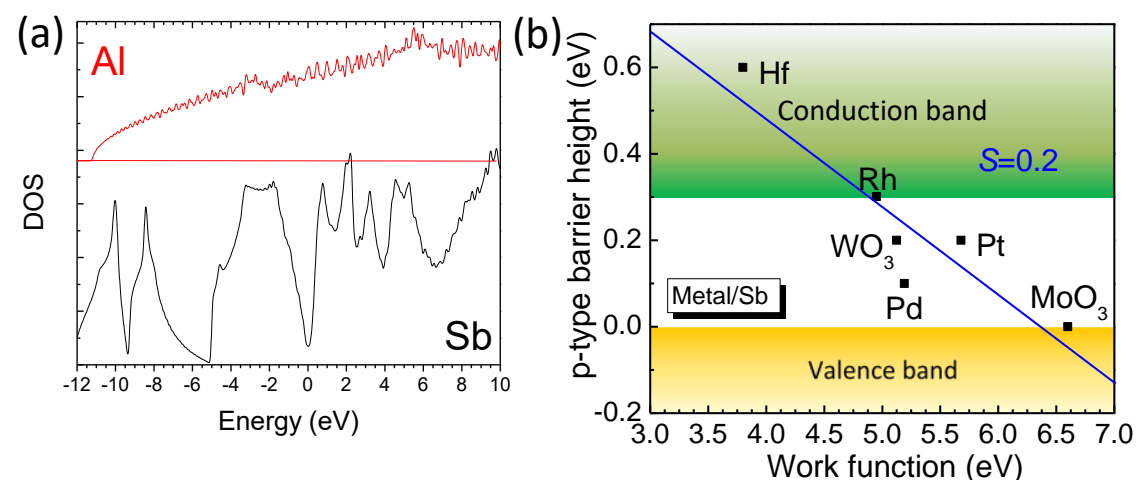

(c)
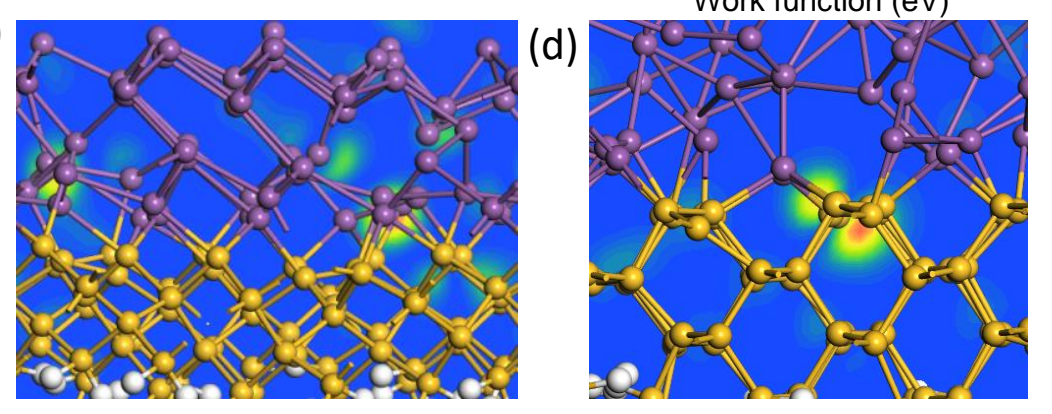

Fig. 10. (a) Density of states of Sb compared to that of the nearly free electron metal Al, to show the small DOS of Sb at its Fermi level $(0 \mathrm{eV})$. (b) Calculated Schottky barrier heights versus metal work functions for various metals on $\mathrm{Sb}$. (c) $\mathrm{Sb}$ on $\mathrm{Si}(100)$, and (d) $\mathrm{Sb}$ on $\mathrm{Si}$ (110) interfaces, showing the wavefunctions at Fermi energy, and the localized 'defect states'. The Si slabs are terminated by Si-H bonds at the bottom. 

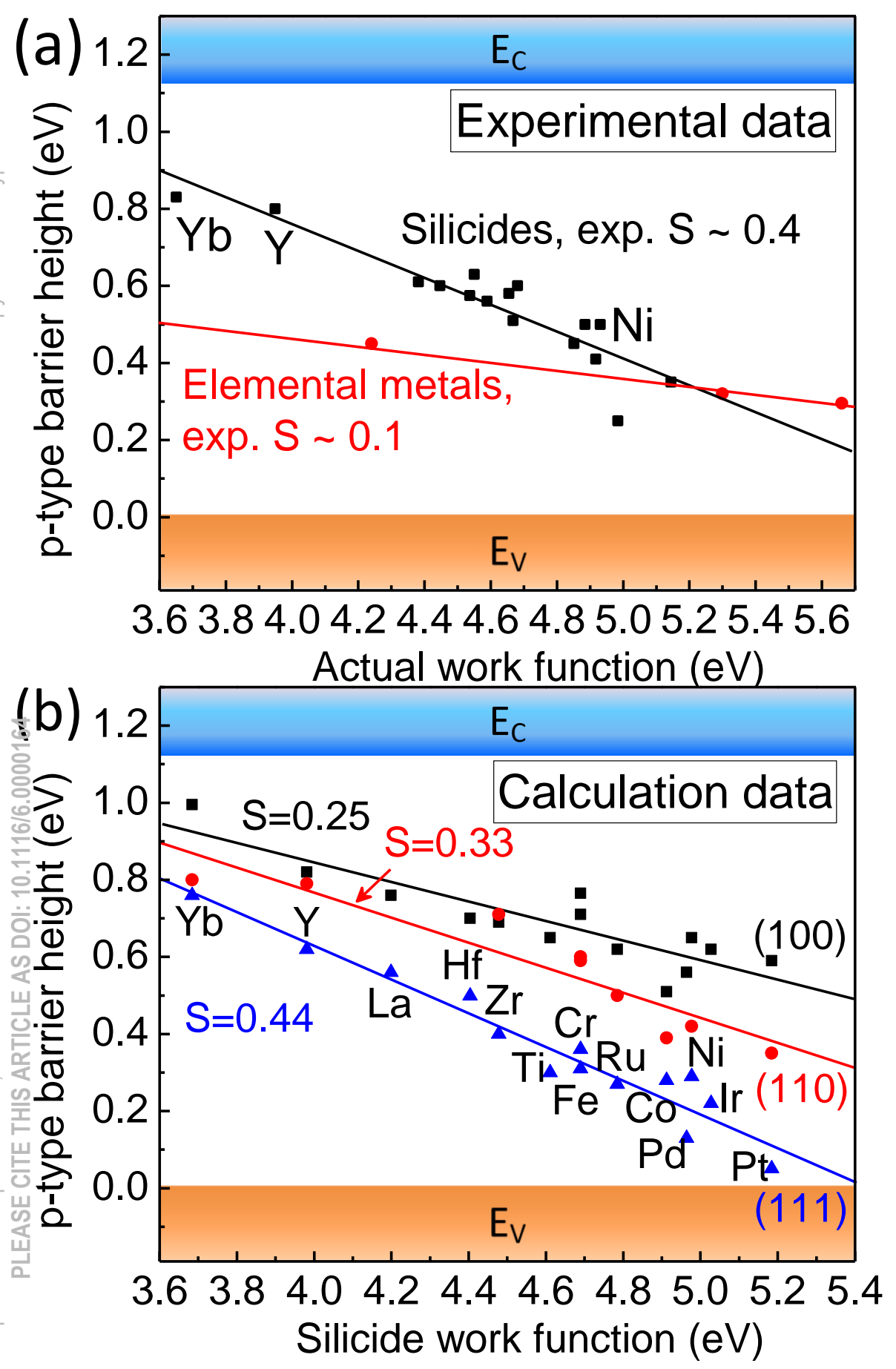

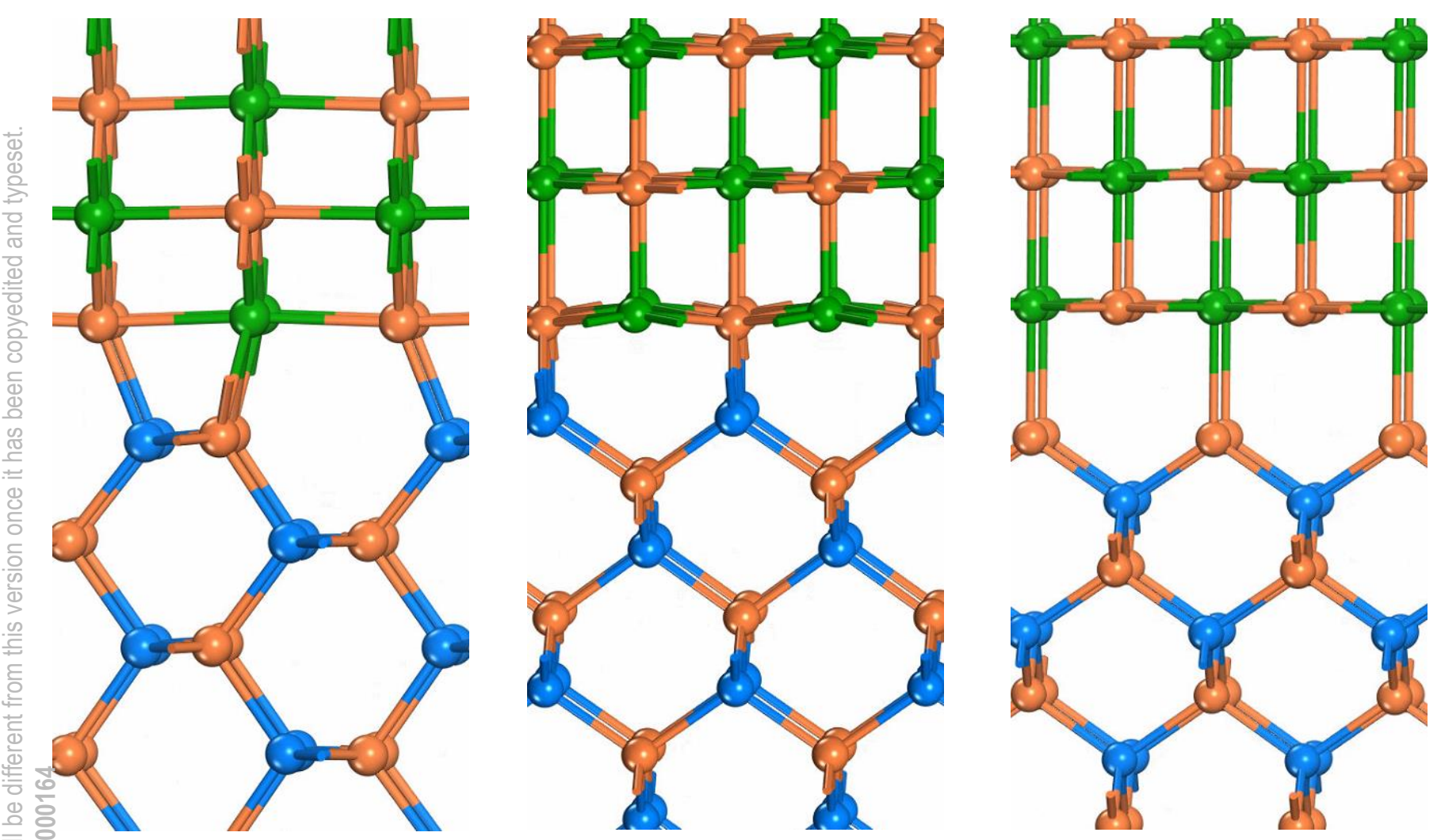

As
Y
Ga

(a) (110) interface (b) (100) Ga-term. (c) (100) As-term.

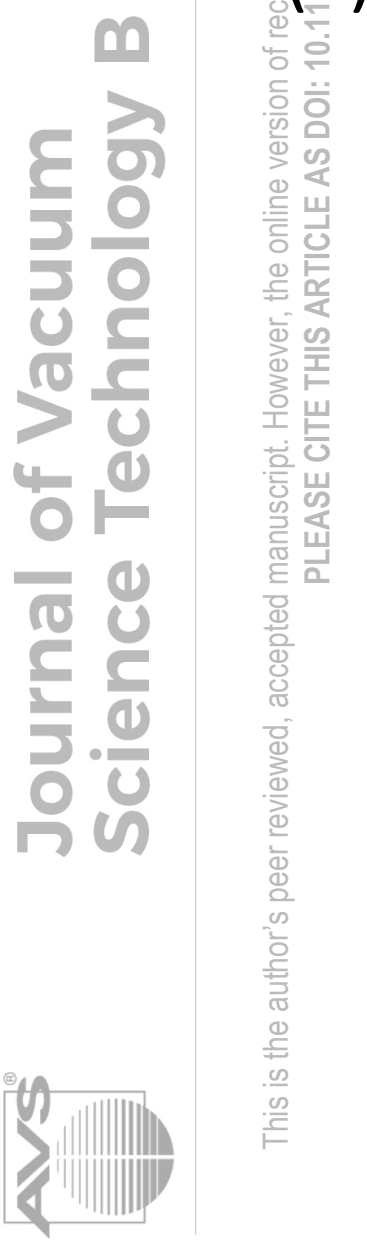



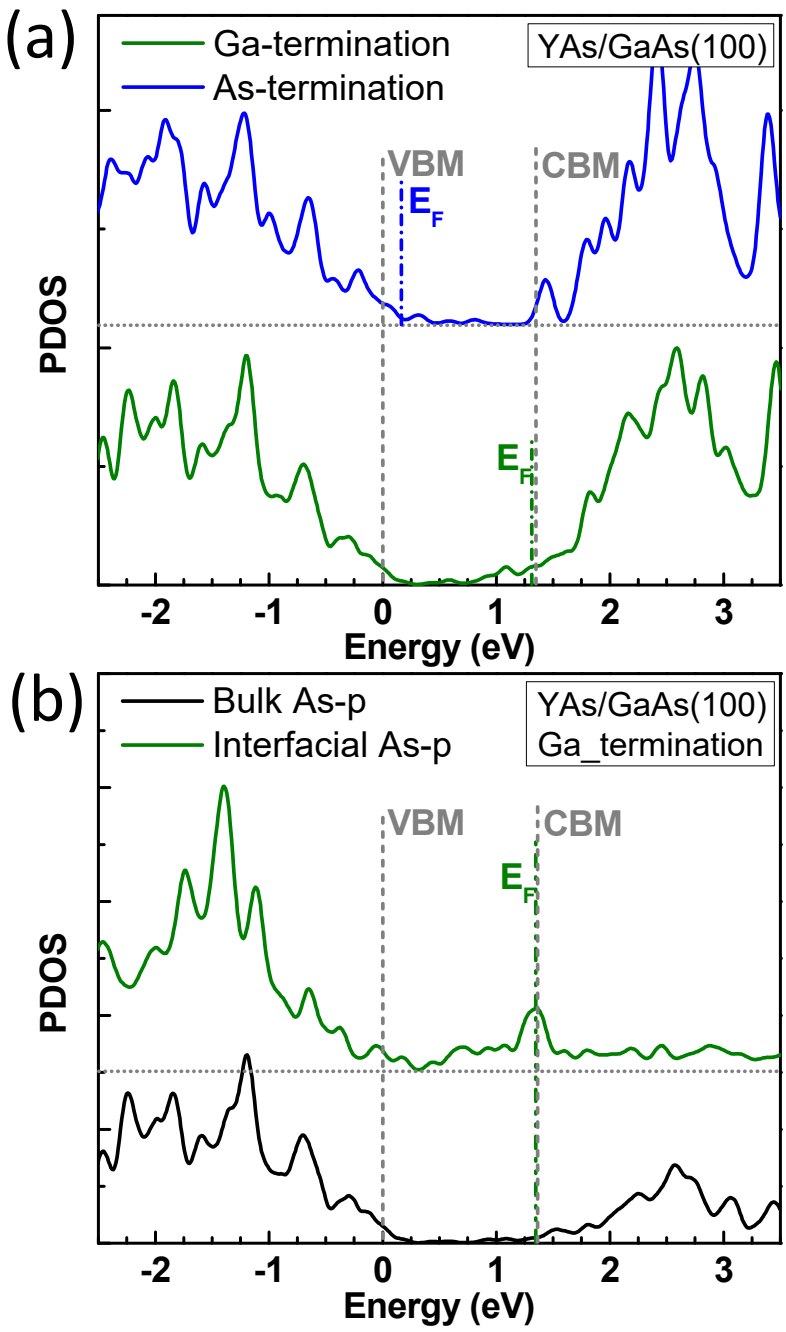

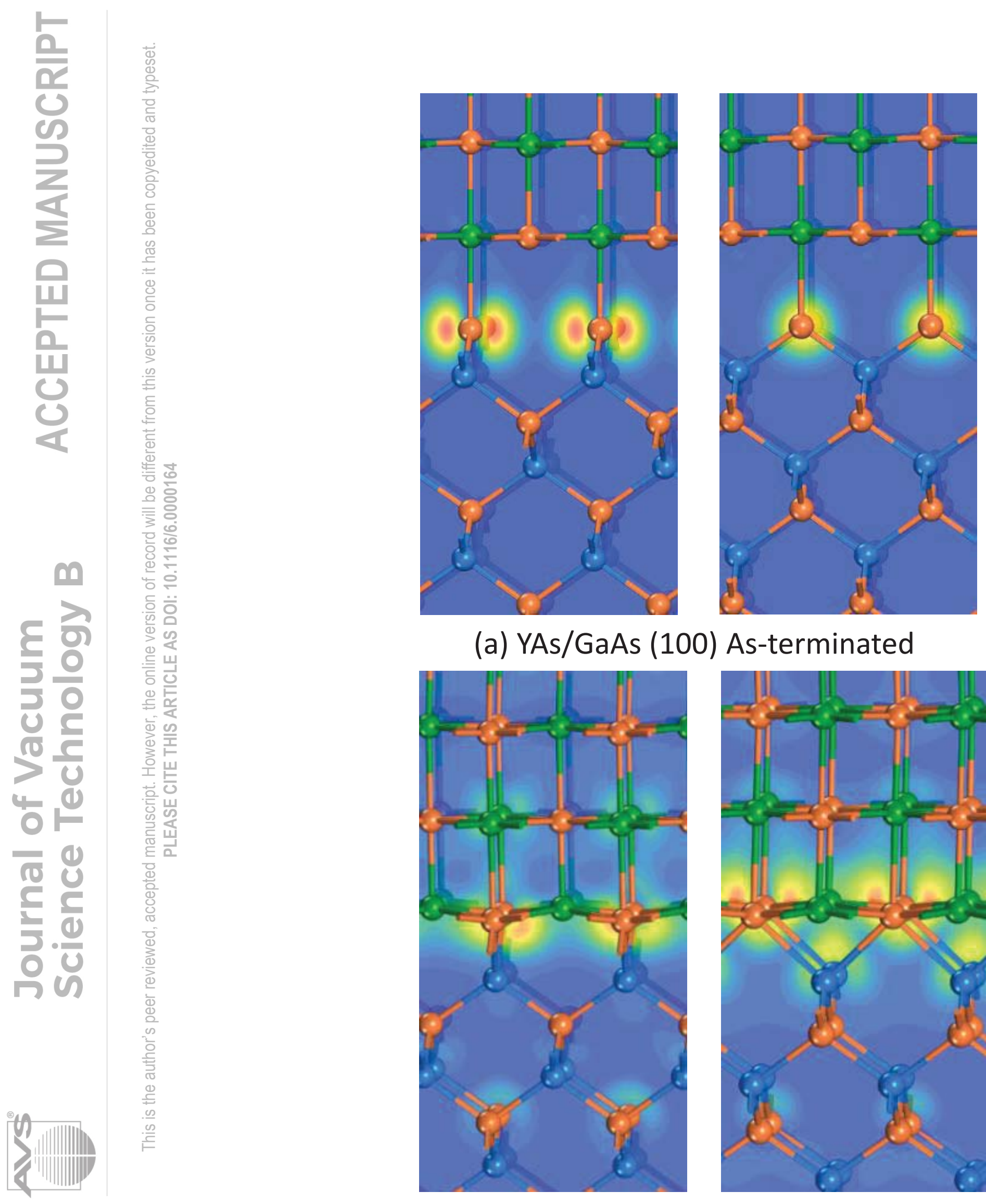

(a) YAs/GaAs (100) As-terminated
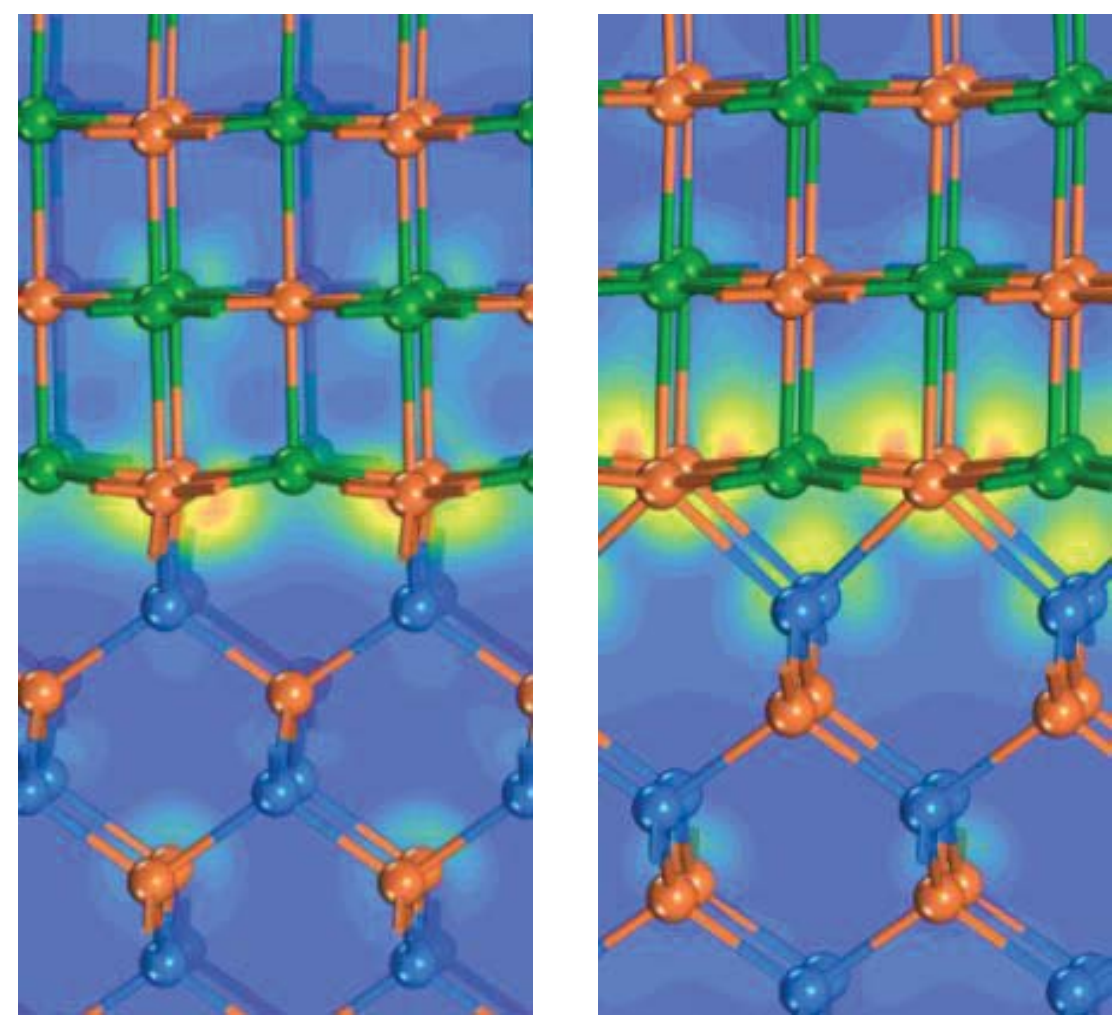

(b) YAs/GaAs (100) Ga-terminated 


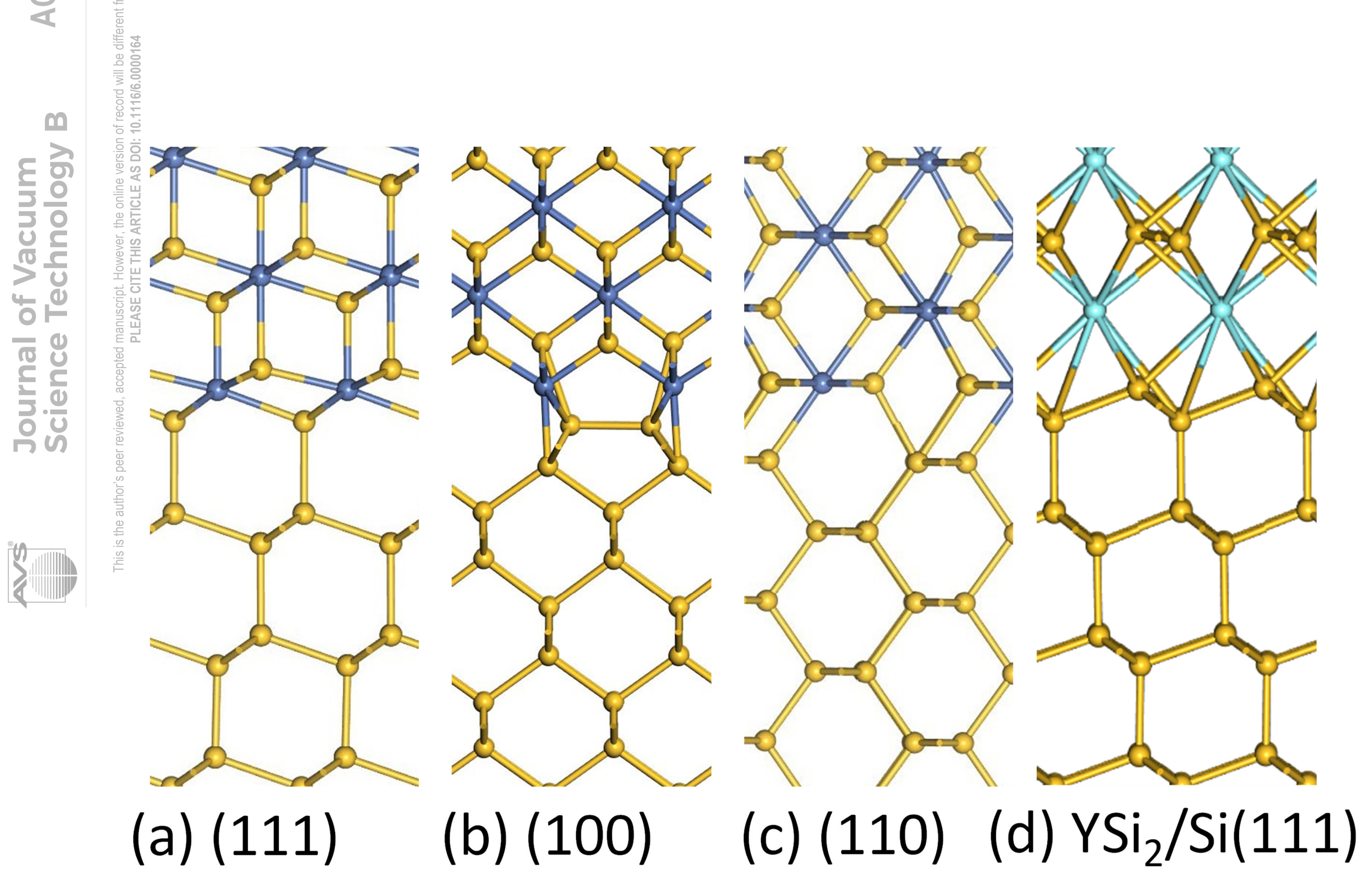




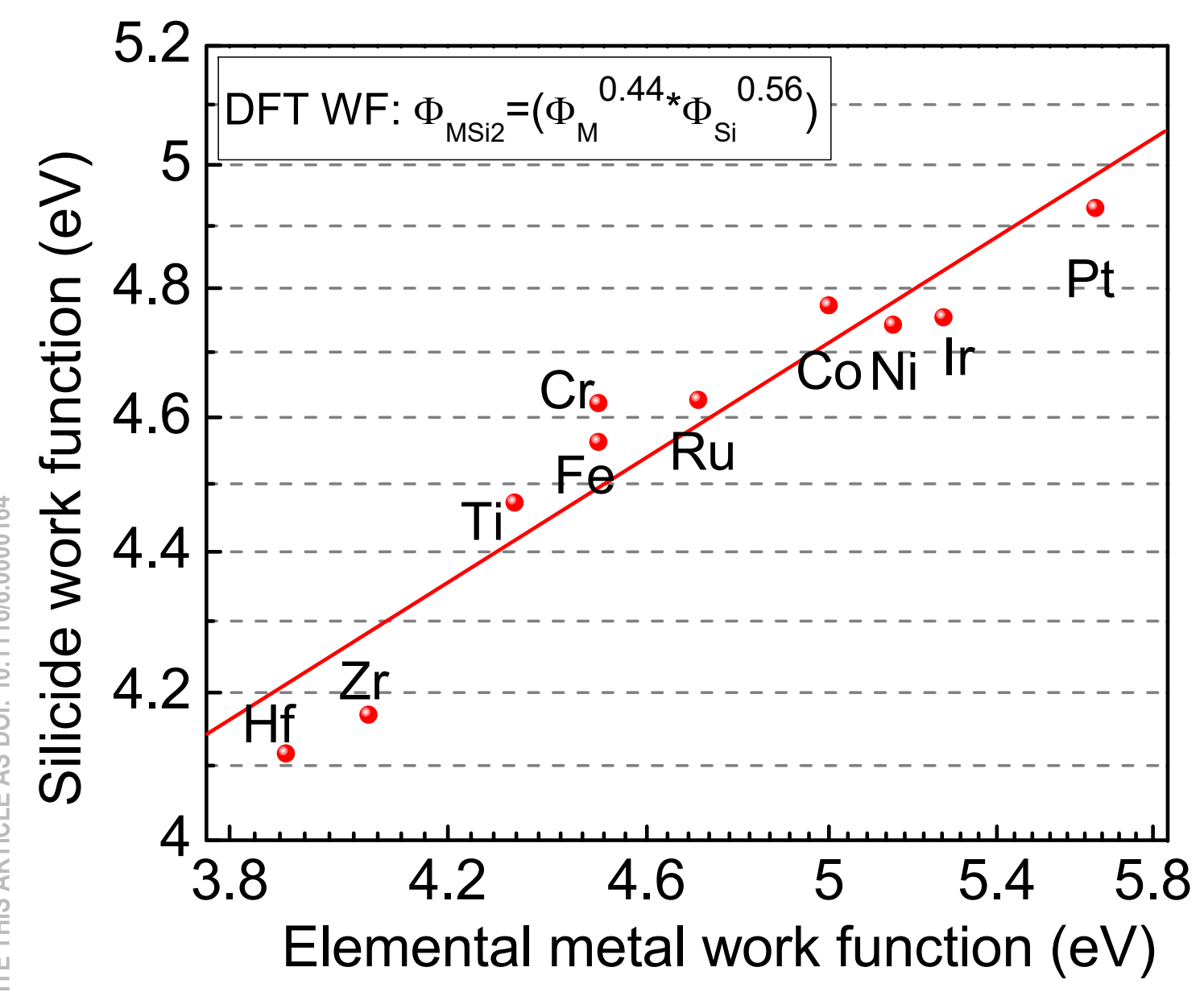




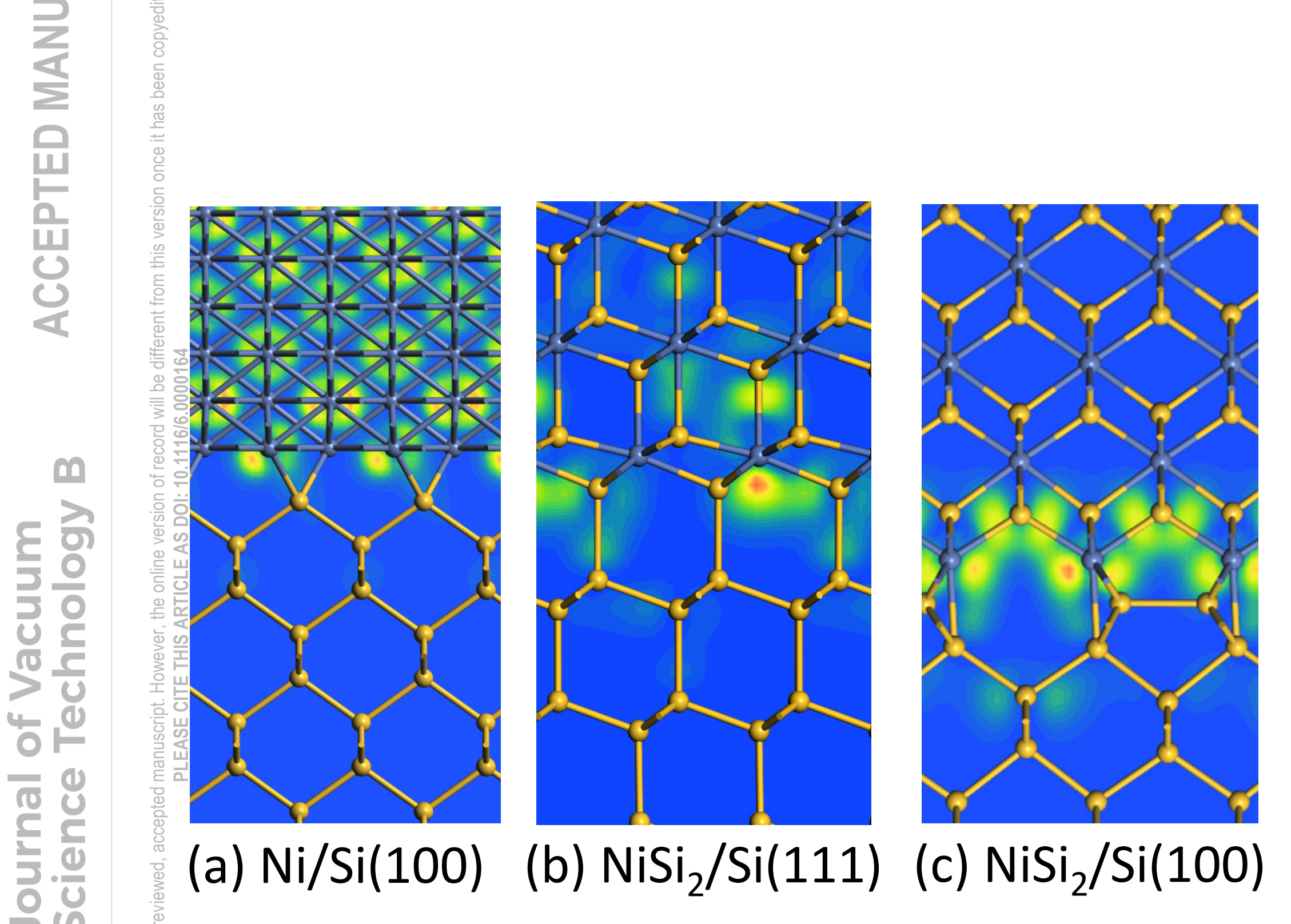




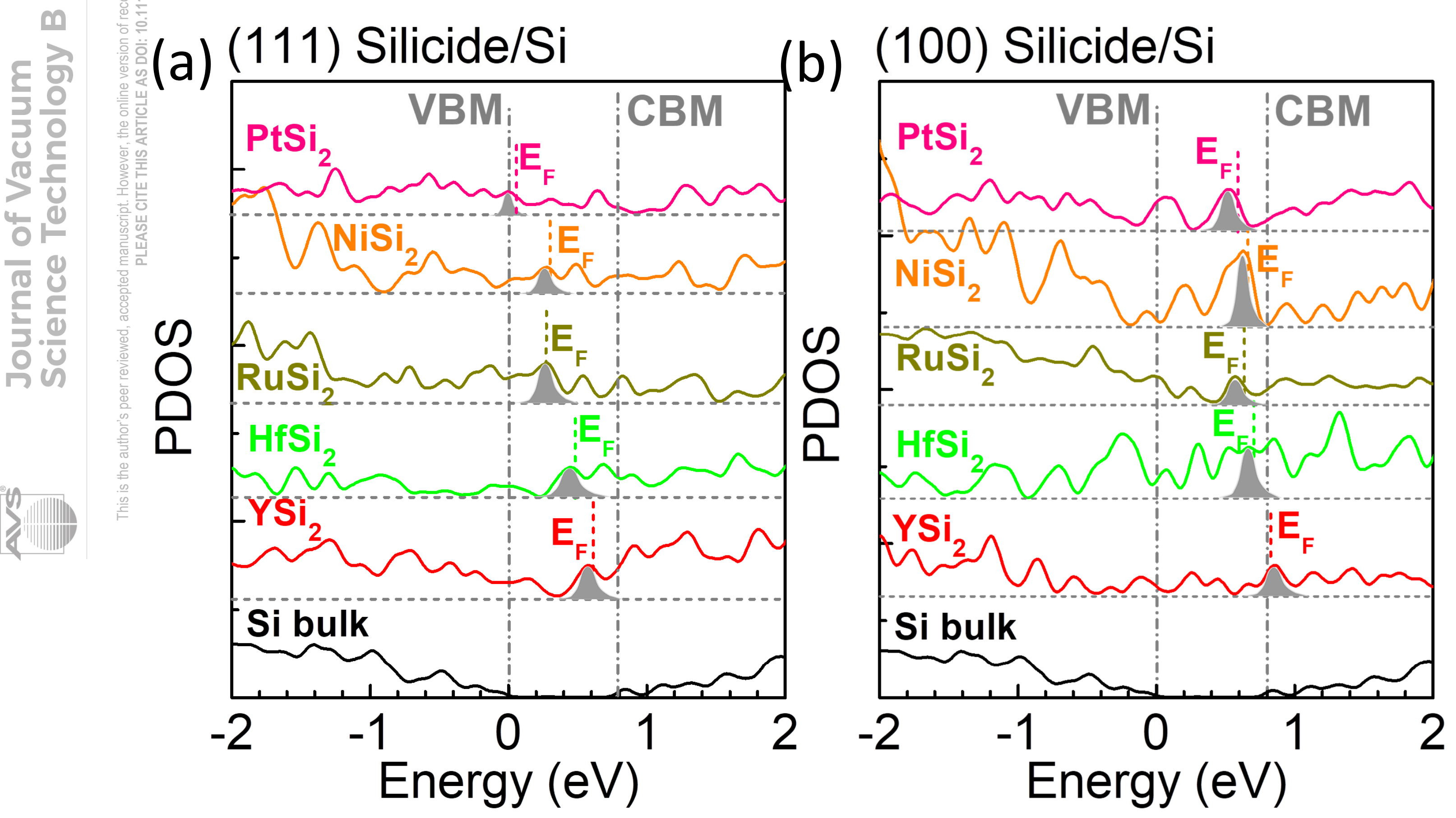




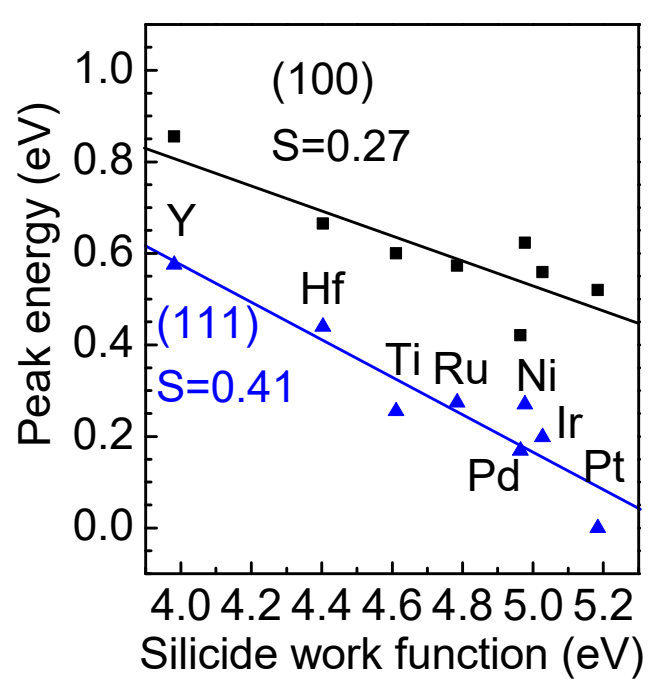




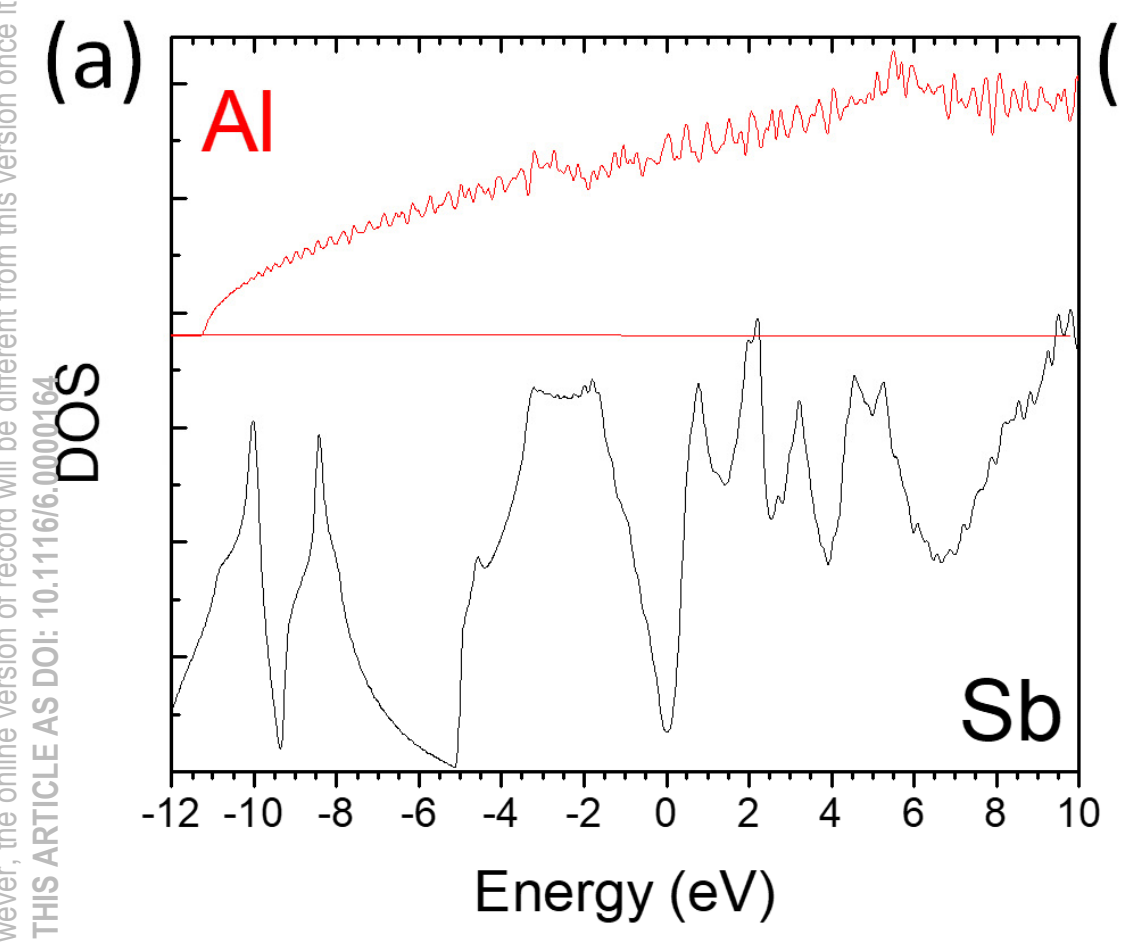

(b)

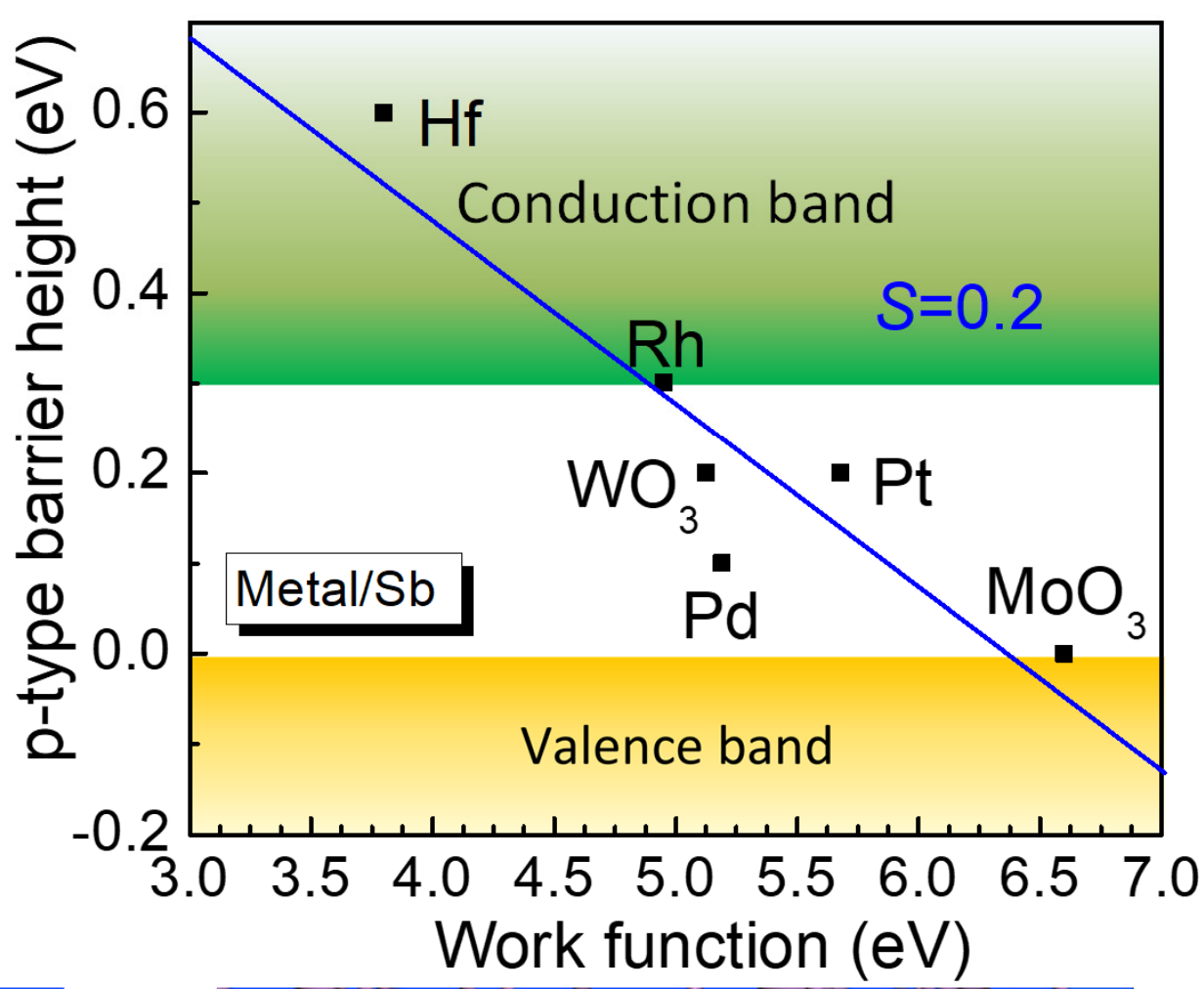

(c)

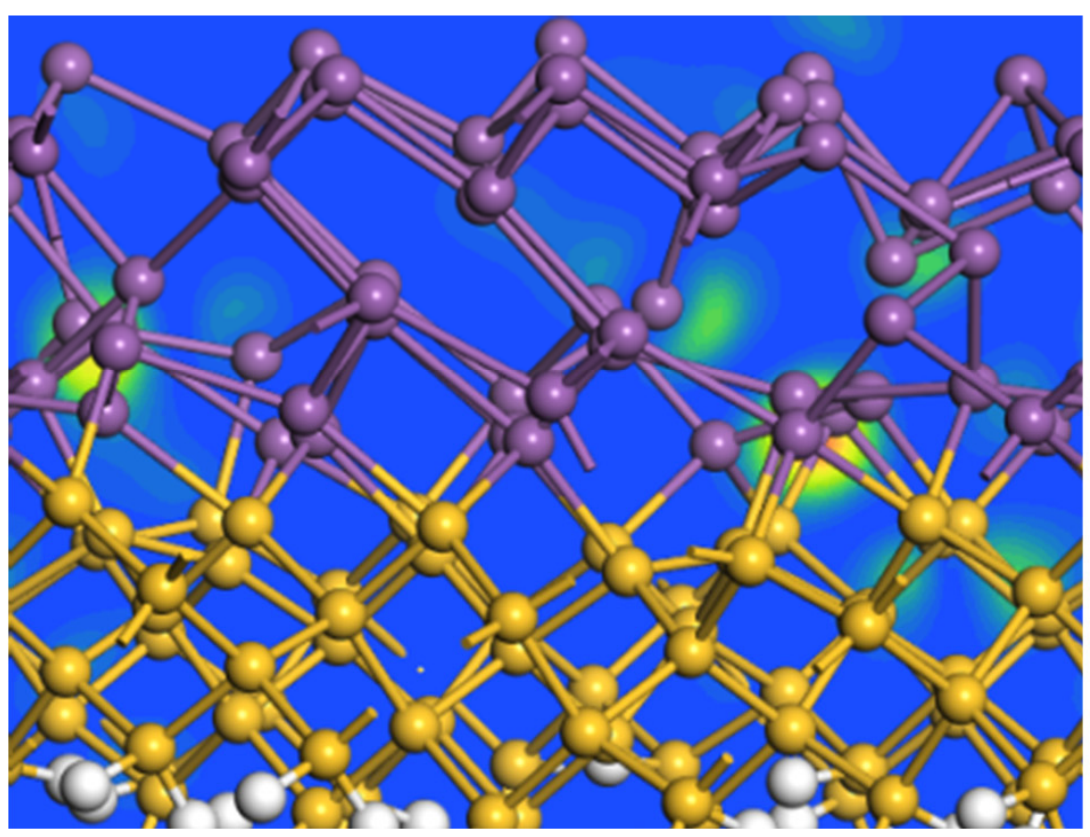

(d)

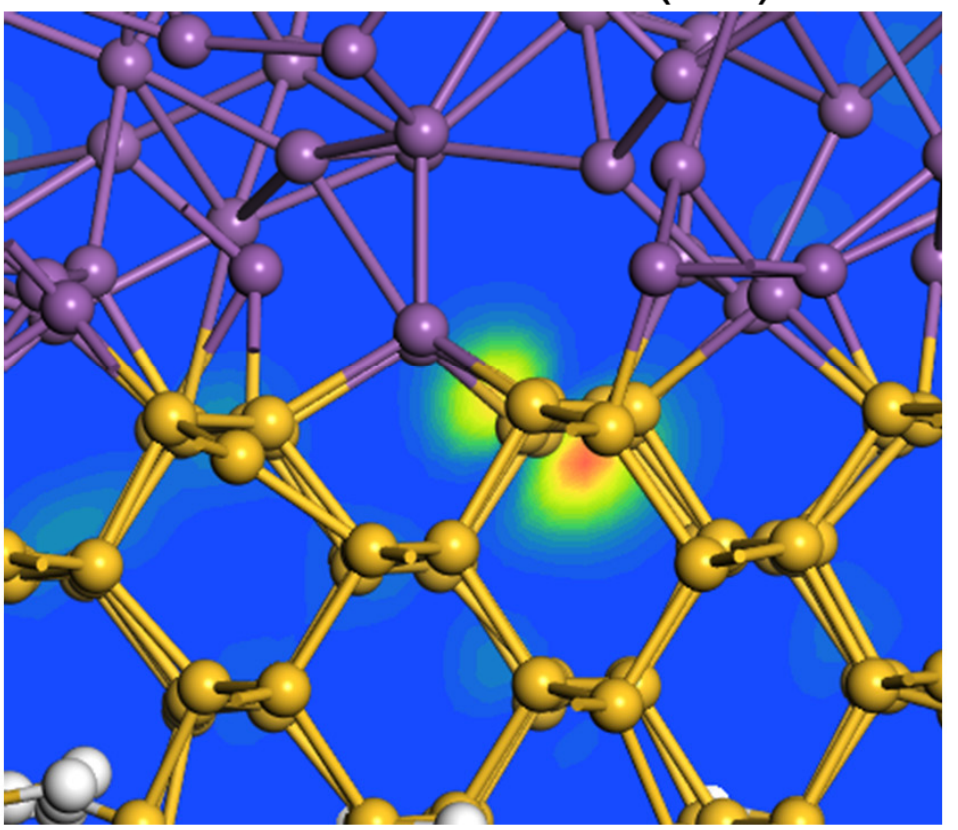

